\title{
Sur les Bathynella de Roumanie: B.(B.) boteai Serban, B.(B.) vaducrisensis N.Sp., B.(B.) plesai Serban et B.(B.) motrensis Serban
}

(Bathynellacea, Bathynellidae)

Première partie

par

Eugène SERBAN

\section{SUMMARY}

On the Bathynella from Romania: B.(B.) boteai Serban, B.(B.) vaducrisensis N.Sp., B.(B.) plesai Serban and B.(B.) motrensis Serban (Bathynellacea, Bathvnellidae). Part I.

In the first part of the note we present a minute description of the species B.(B.) boteai Serban, B.(B.) motrensis Serban and B.(B.) plesai Serban. The provisional diagnosis of thes species were already published in 1971. A new species B.(B.) vaducrisensis is also described. The genus Bathynella Vejdovsky is now known in the Romanian fauna with 6 species: four of them already mentioned, plus B.(B.) paranatans Serban and B.(B.) scythica Botosaneanu and Damian.

\section{INTRODUCTION}

Suite à nos contributions initiales sur la taxonomie du genre Bathynella (Serban, 1966, 1966a, 1970), nous avons décrit quatre espèces nouvelles de Roumanie: B.(B.) paranatans, B.(B.) boteai, B.(B.) motrensis et B.(B.) plesai (Serban, 1971). L'espèce $B$.(B.) paranatans, dont la morphologie externe a été présentée exhaustivement dans le travail intitulé "Bathynella" (Serban, 1972), est devenue le taxon le mieux connu du genre, sa description venant compenser, provisoirement, la connaissance trop sommaire de l'espèce type du genre, $B$. natans Vejd. D'une part, ces premiers résultats ont permis la mise en évidence des limites de la structuration dans le genre Bathynella et, d'autre part, ont conduit à une nouvelle conception taxonomique. Si, auparavant, le genre Bathynella était tenu comme un élément qui peuple le monde entier, aujourd'hui on sait qu'il n'est pas le seul d'Europe (Serban, Coineau et Delamare Deboutteville, 1971, 1972; Serban, 1973, 1973a) et que sa présence en Amérique australe, soutenue par certains auteurs, n’a pas été encore

\footnotetext{
* Institut de Spéologie “Emile Racovitza”, 9, rue Mihail Moxa, Bucarest. Roumanie.
} 
prouvée (Delamare Deboutteville et Serban, 1973); notons aussi que l'appartenance des espèces d'Asie et d'Australie au genre Bathynella est également sujette à caution.

Dans la présente note, complément à nos recherches sur les Bathynella de Roumanie, nous donnons la description détaillée des espèces $B$.(B.) boteai, (B.(B.) plesai et $B$.(B.) motrensis; rappelons que leurs diagnoses préliminaires ont été publiées dans une contribution ayant comme but principal la discussion des desiderata de la systématique du genre Bathynella (Serban, 1971). Le quatrième taxon qui fait l'objet de cette note, B.(B.) vaducrisensis n.sp., n'est que l'espèce citée dans l'étude de Bathynella (Serban, 1972) comme Bathynella sp.

Nous remercions vivement le Dr. C. Plesa qui, trouvant lors de ses recherches sur la faune souterraine de nombreux Bathynella, a eu l'amitié dé nous les céder pour étude; B.(B.) vaducrisensis n.sp. est la troisième espèce nouvelle découverte par notre collègue.

\section{DESCRIPTION DES ESPECES}

\section{Bathynella (Bathynella) boteai Serban, 1971 (Pl. 1-8)}

Matériel. Les 6 adultes et les 3 individus en cours de développement postembryonnaire proviennent du milieu interstitiel de la rivière de Dragan; les stations prospectées se trouvent à $500 \mathrm{~m}$ du confluent de la rivière de Dragan et la rivière de Crisul Repede, village Valea Draganului (à 4 km de la localité Ciucea), dép. de Cluj, Transylvanie; leg. Fr. Botea, 28.VII.1965. Jusqu'à présent l'espèce n'a pas été retrouvée dans d'autres endroits de la région, bien que le milieu interstitiel des rivières de Dragan et de Crisul Repede ait été bien prospecté. Précisons aussi que les recherches poursuivies par Fr. Botea et C. Plesa le long de la rivière de Dragan ont eu comme résultat la découverte de plusieurs stations peuplées par B.(B.) paranatans, espèce trouvée initialement dans le milieu interstitiel de la rivière de Crisul Repede.

Taille. L'espèce dépasse $1 \mathrm{~mm}$ en longueur, certaines femelles atteignant $1,5 \mathrm{~mm}$ ou même $1,8 \mathrm{~mm}$. Etant donné cette taille, B.(B.) boteai eșt le représentant le plus robuste du genre.

Antennule (P1. 1 A). Cet appendice diffère de celui de B.(B.) paranatans par l'absence du poil interne et proximal de l'article basal du pédoncule (voir le poil a1, P1. $\mathrm{X}$, in Serban, 1972).

Antenne (Pl. 1 B.C). Les traits les plus caractéristiques de l'antenne sont la taille du deuxième article endopodial et la chétotaxie de l'exopodite. Si chez B.(B.) paranatans l'article endopodial. II est plus court que le cinquième, chez B.(B.) boteai tous les deux ont la même longueur; vu ce trait, l'antenne, prise dans sa totalité, égale la longueur de l'antennule. L'exopodite, de la même longueur que l'article endopodial III, ne porte pas le poil médian que l'on trouve toujours chez B.(B.) paranatans.

Mandibule (P1. 2). Bien que nous ayons eu à notre disposition seulement 6 indi- 


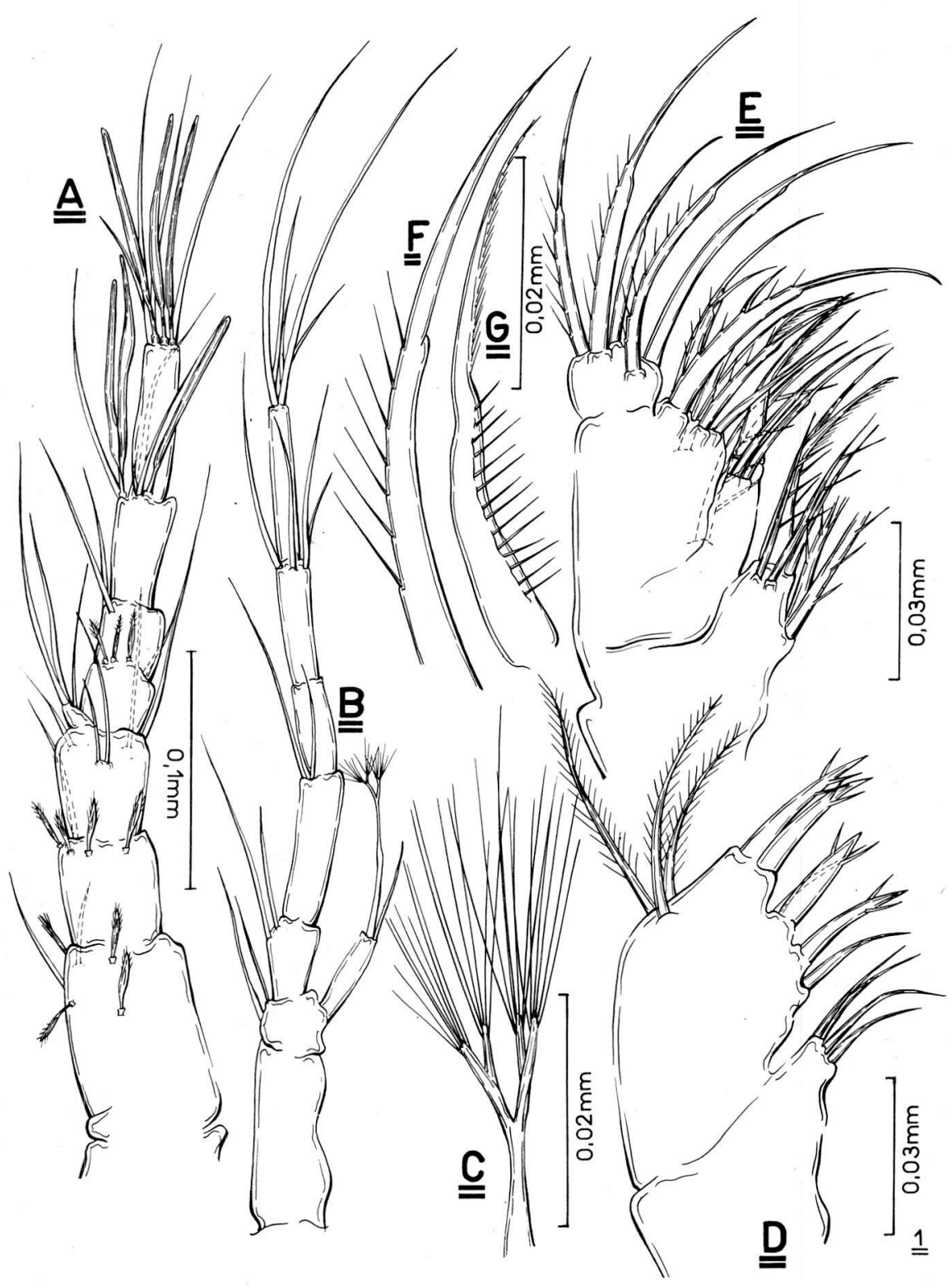

Planche 1. Bathynella (Bathynella) boteai Serban. A, antennules; B, antenne; C, ramification apicale de l'organe sensoriel de l'antenne; D, maxillule; E, maxille; F, poil endopodial cilié sur le bord externe (maxille); $\mathrm{G}$, griffe maxillaire. 

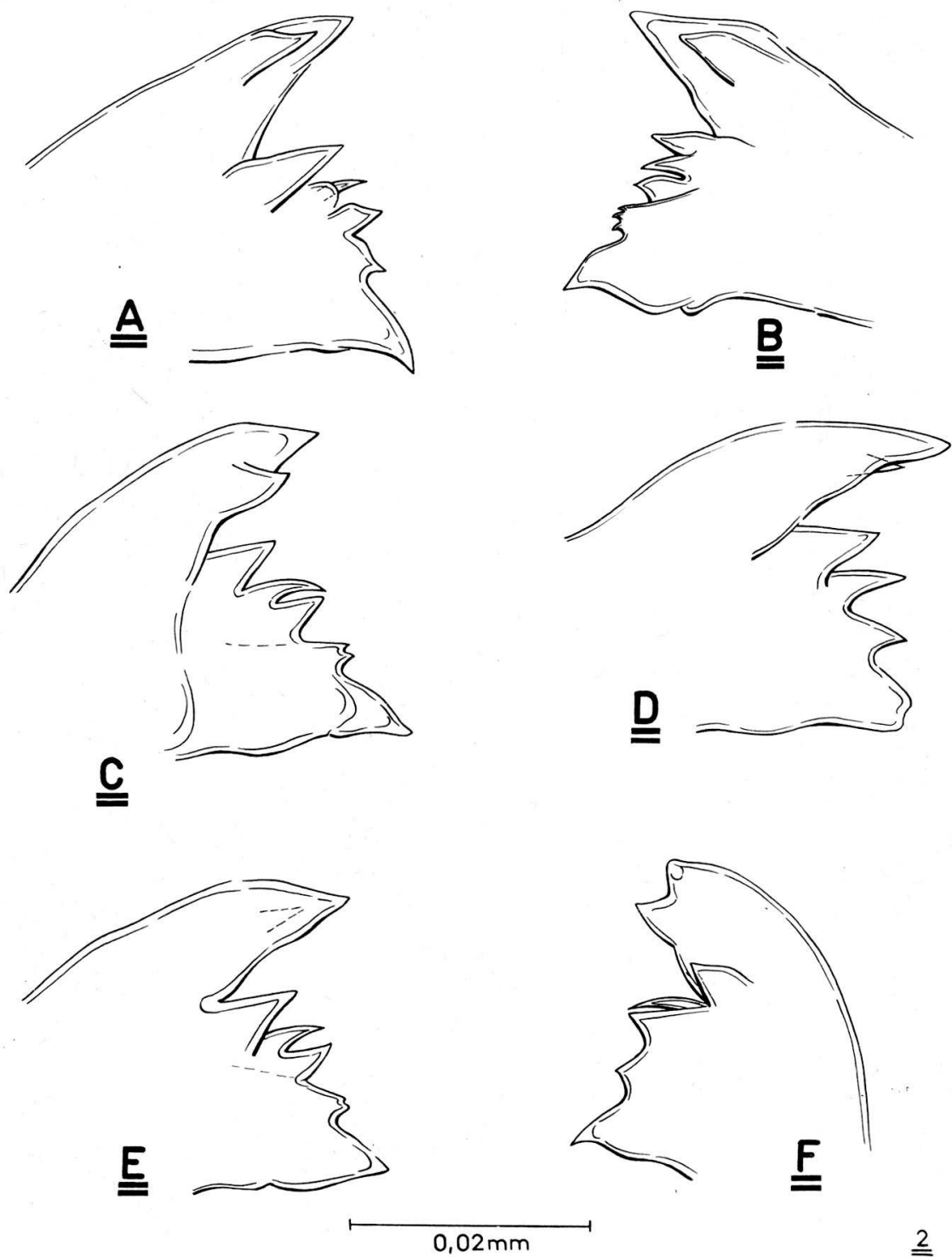

Planche 2. Bathynella (Bathynella) boteai Serban. Partie masticatrice des mandibules: A, femelle; B, mâle; C,D, femelle; E,F, femelle. 
vidus, la partie masticatrice de la mandibule, à structure générale typique, présente, chez certains exemplaires, un degré de variabilité de l'avant-dernière dent: s1 chez l'une des mandibules cette dent conserve la forme habituelle, chez l'autre elle peut porter 2 ou 3 denticules.

Maxillule (Pl. 1 D). Dans la première description de l'espèce, nous avons mentionné que les 4 dents de cette pièce buccale sont des dents maxillulaires à épines (chez B.(B.) paranatans on n'en trouve que trois pareilles, la quatrième étant une dent maxillulaire glabre). Chez les femelles, l'une des dents apicales porte 3 épines sur l'une des maxillules et 2 sur l'autre; chez les mâles, toutes les dents ont 2 épines.

Maxille (Pl. 1 E,F,G). Bien que le nombre des phanères et la structure générale de cet appendice soient identiques à ceux de B.(B.) paranatans, le palpe porte 3 poils endopodiaux ciliés sur le bord externe et 2 poils endopodiaux simples; chez $B$. (B.) paranatans, tous les 5 phanères du palpe sont des poils endopodiaux simples.

Péréiopodes I-VII (P1. 3-5). Première paire sans épipodite respiratoire; vu de face, l'exite précoxal des paires antérieurs a un contour général rectangulaire; exopodite de tous les péréiopodes à 5 poils; éperon coxal de la paire VII du mậle, allongé $(\mathrm{Pl} .5 \mathrm{C})$. En ajoutant aux trois premières caractéristiques, qui ne se rencontrent jamais chez B.(B.) paranatans, le nombre nettement plus élevé de poils sur la plupart des péréiopodes, on voit que la morphologie des appendices ambulatoires présente une large série de particularités.

Lors de l'étude de $B$.(B.) paranatans, nous avons précisé que les combinaisons numériques les plus fréquentes de la chétotaxie ont respectivement 42, 32, 32, 30 , $26,18,16$ poils pour les péréiopodes I-VII des mâles et $44,34,32,32,30,18$, 16 poils pour ceux des femelles. Si l'on compare maintenant les combinaisons numériques les plus complètes dans les deux sexes de B.(B.) boteai (tableaux 1 et 2 ) à celles de $B .(B$.) paranatans (tableaux 3 et 4 ), il est à souligner les remarques suivantes*:

a) tous les péréiopodes de $B$.(B.) boteai portent un nombre plus grand de poils que ceux de $B$.(B.) paranatans, cette différence numérique étant plus marquée chez les quatre premières paires; ce fait est dû à la chétotaxie plus riche des articles endopodiaux IV portant 4 phanères sur les péréiopodes I-IV, à celle des autres articles endopodiaux (péréiopodes I-VI) et du basipodite (paires I-IV),ainsi qu'à la chétotaxie de l'exopodite des appendices VII, composée de 5 poils;

b) contrairement à la combinaison numérique fondamentale de la chétotaxie des péréiopodes de $B$. (B.) paranatans ayant comme élément le plus fréquent le nombre de 2 poils - 0/0 2/2 2/2 2/2 2/2 3/3 5/5, la combinaison de $B$.(B.) boteai a le même élément représenté par le nombre de 3 poils - $0 / 03 / 33 / 33 / 33 / 34 / 45 / 5$;

c) pour les mâles et respectivement pour les femelles de B.(B.) paranatans, la combinaison numérique fondamentale est présente sur les péréiopodes II-III et III-IV; chez B.(B.) boteai, on la trouve sur les paires II-III des mâles et II-IV des femelles;

* Certaines de ces remarques ont une valeur relative car le nombre d'exemplaires étudiés est assez réduit. 
d) bien que le nombre de poils de la première paire de $B$.(B.) boteai soit plus grand de 12 (mâles) et 10 (femelles) que celui de $B .(B$.$) paranatans, les péréiopodes$ VII portent seulement 2 poils de plus, différence due à la chétotaxie des exopodites; il ressort donc, que la réduction numérique des poils dans le cadre de la série de 7 péréiopodes est plus accusée chez B.(B.) boteai, réduction déterminant une chétotaxie bien semblable sur les derniers péréiopodes des deux espèces. Analysons plus minutieusement ce processus.

Tableau 1. Bathynella (Bathynella) boteai Serban Combinaisons numériques de la chétotaxie des péréiopodes I-VII (mâle)

\begin{tabular}{llllllllc}
\hline Péréiopỏe & Cx & Bsp & I & II & III & IV & Exp & $\begin{array}{c}\text { Nombre total } \\
\text { de poils }\end{array}$ \\
\hline I & $1 / 1$ & $5 / 5$ & $5 / 5$ & $4 / 4$ & $3 / 3$ & $4 / 4$ & $5 / 5$ & 54 \\
II & $0 / 0$ & $3 / 3$ & $3 / 3$ & $3 / 3$ & $3 / 3$ & $4 / 4$ & $5 / 5$ & 42 \\
III & $0 / 0$ & $3 / 3$ & $3 / 3$ & $3 / 3$ & $3 / 3$ & $4 / 4$ & $5 / 5$ & 42 \\
IV & $0 / C$ & $3 / 3$ & $3 / 3$ & $3 / 3$ & $2 / 2$ & $4 / 4$ & $5 / 5$ & 40 \\
V & $0 / 0$ & $1 / 1$ & $2 / 2$ & $2 / 2$ & $2 / 2$ & $3 / 3$ & $5 / 5$ & 30 \\
VI & $0 / 0$ & $1 / 1$ & $2 / 2$ & $0 / 0$ & $0 / 0$ & $2 / 2$ & $5 / 5$ & 20 \\
VII & $0 / 0$ & $1 / 1$ & $1 / 1$ & $0 / 0$ & $0 / 0$ & $2 / 2$ & $5 / 5$ & 18
\end{tabular}

Tableau 2. Bathynella (Bathynella) boteai Serban

Combinaisons numériques de la chétotaxie des péréiopodes I-VII (femelle)

\begin{tabular}{lcccccccc}
\hline Péréiopode & Cx & Bsp & I & II & III & IV & Exp. & $\begin{array}{c}\text { Nombre total } \\
\text { de poils }\end{array}$ \\
\hline I & $1 / 1$ & $5 / 5$ & $5 / 5$ & $4 / 4$ & $3 / 3$ & $4 / 4$ & $5 / 5$ & 54 \\
II & $0 / 0$ & $3 / 3$ & $3 / 3$ & $3 / 3$ & $3 / 3$ & $4 / 4$ & $5 / 5$ & 42 \\
III & $0 / 0$ & $3 / 3$ & $3 / 3$ & $3 / 3$ & $3 / 3$ & $4 / 4$ & $5 / 5$ & 42 \\
IV & $0 / 0$ & $3 / 3$ & $3 / 3$ & $3 / 3$ & $3 / 3$ & $4 / 4$ & $5 / 5$ & 42 \\
V & $0 / 0$ & $2 / 2$ & $2 / 2$ & $2 / 2$ & $2 / 2$ & $3 / 3$ & $5 / 5$ & 32 \\
VI & $0 / 0$ & $1 / 1$ & $2 / 2$ & $0 / 0$ & $0 / 0$ & $2 / 2$ & $5 / 5$ & 20 \\
VII & $0 / 0$ & $1 / 1$ & $1 / 1$ & $0 / 0$ & $0 / 0$ & $2 / 2$ & $5 / 5$ & 18
\end{tabular}


Tableau 3. Bathynella (Bathynella) paranatans Serban Combinaisons numériques de la chétotaxie des péréiopodes I-VII (mâle) (d'après Serban, 1972)

\begin{tabular}{lcccccccc}
\hline Péréiopode & Cx & Bsp & I & II & III & IV & Exp & $\begin{array}{c}\text { Nombre total } \\
\text { de poils }\end{array}$ \\
\hline I & $1 / 1$ & $3 / 3$ & $4 / 4$ & $3 / 3$ & $2 / 2$ & $3 / 3$ & $5 / 5$ & 42 \\
II & $0 / 0$ & $2 / 2$ & $2 / 2$ & $2 / 2$ & $2 / 2$ & $3 / 3$ & $5 / 5$ & 32 \\
III & $0 / 0$ & $2 / 2$ & $2 / 2$ & $2 / 2$ & $2 / 2$ & $3 / 3$ & $5 / 5$ & 32 \\
IV & $0 / 0$ & $1 / 1$ & $2 / 2$ & $2 / 2$ & $2 / 2$ & $3 / 3$ & $5 / 5$ & 30 \\
V & $0 / 0$ & $1 / 1$ & $2 / 2$ & $1 / 1$ & $1 / 1$ & $3 / 3$ & $5 / 5$ & 26 \\
VI & $0 / 0$ & $1 / 1$ & $1 / 1$ & $0 / 0$ & $0 / 0$ & $2 / 2$ & $5 / 5$ & 18 \\
VII & $0 / 0$ & $1 / 1$ & $1 / 1$ & $0 / 0$ & $0 / 0$ & $2 / 2$ & $4 / 4$ & 16
\end{tabular}

Tableau 4. Bathynella (Bathynella) paranatans Serban Combinaisons numériques de la chétotaxie des péréiopodes I-VII (femelle) (d'après Serban, 1972)

\begin{tabular}{lcccccccc}
\hline Péréiopode & Cx & Bsp & I & II & III & IV & Exp & $\begin{array}{c}\text { Nombre total } \\
\text { de poils }\end{array}$ \\
\hline I & $1 / 1$ & $3 / 3$ & $4 / 4$ & $3 / 3$ & $3 / 3$ & $3 / 3$ & $5 / 5$ & 44 \\
II & $0 / 0$ & $2 / 2$ & $3 / 3$ & $2 / 2$ & $2 / 2$ & $3 / 3$ & $5 / 5$ & 34 \\
III & $0 / 0$ & $2 / 2$ & $2 / 2$ & $2 / 2$ & $2 / 2$ & $3 / 3$ & $5 / 5$ & 32 \\
IV & $0 / 0$ & $2 / 2$ & $2 / 2$ & $2 / 2$ & $2 / 2$ & $3 / 3$ & $5 / 5$ & 32 \\
V & $0 / 0$ & $1 / 1$ & $2 / 2$ & $2 / 2$ & $2 / 2$ & $3 / 3$ & $3 / 3$ & 30 \\
VI & $0 / 0$ & $1 / 1$ & $1 / 1$ & $0 / 0$ & $0 / 0$ & $2 / 2$ & $5 / 5$ & 18 \\
VII & $0 / 0$ & $1 / 1$ & $1 / 1$ & $0 / 0$ & $0 / 0$ & $2 / 2$ & $4 / 4$ & 16
\end{tabular}

Chez B.(B.) paranatans, on distingue, selon le nombre de poils des diverses paires, trois catégories de péréiopodes qui diffèrent entre elles par environ 10 phanères: les paires I, II-V et VI-VII. Entre la chétotaxie des péréiopodes appartenant au même groupe les différences sont moins accentuées ou nulles, ayant une valeur maximale de 2 poils. Chez B.(B.) boteai, on trouve quatre catégories semblables: les péréiopodes I, II-IV, V et VI-VII; dans ce cas, la cinquième paire se détache au point de vue numérique des trois autres précédentes, ayant 10 poils de moins 


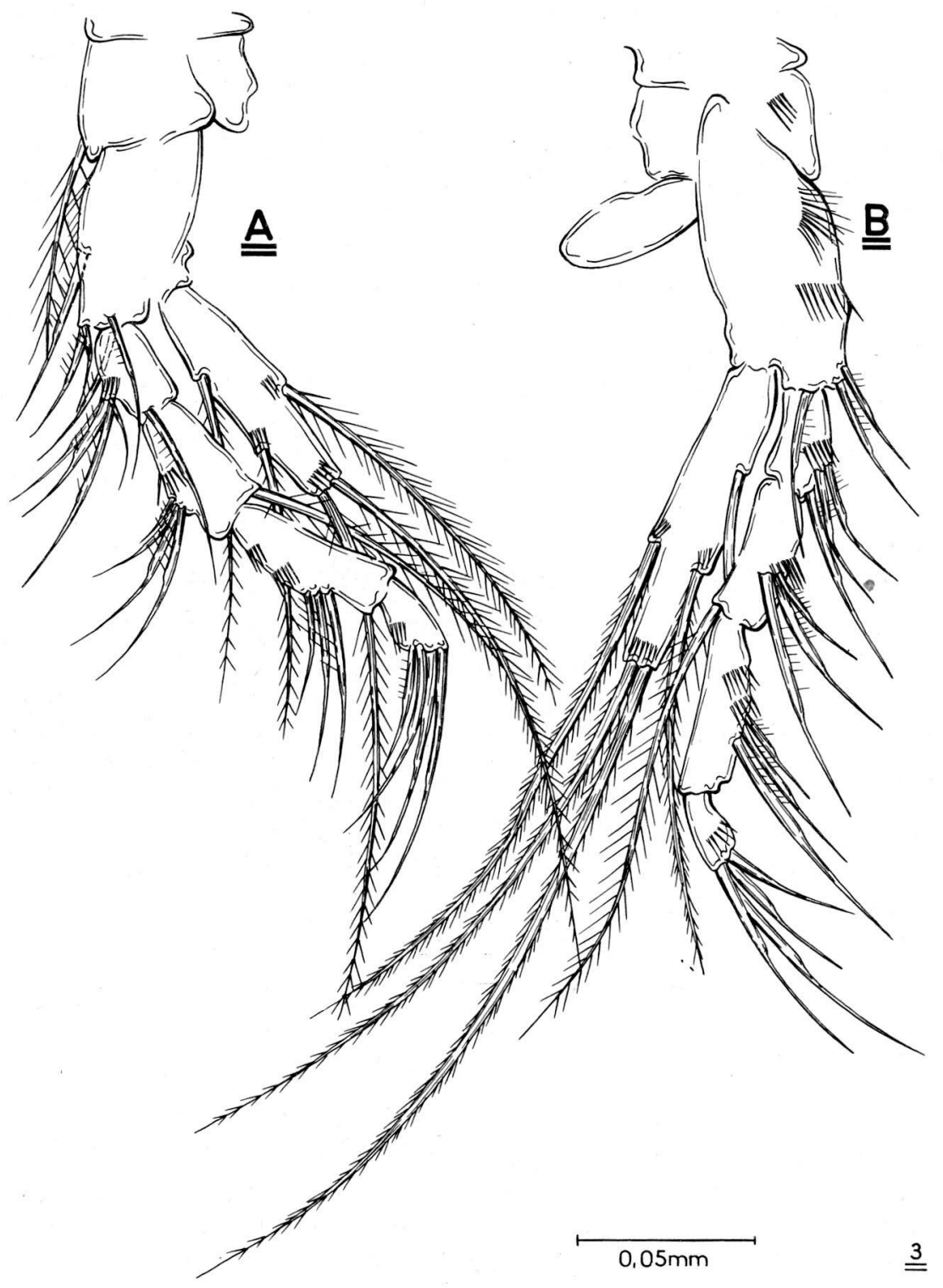

Planche 3. Bathynella (Bathynella) boteai Serban. A, péréiopode I; B, péréiopode II. 


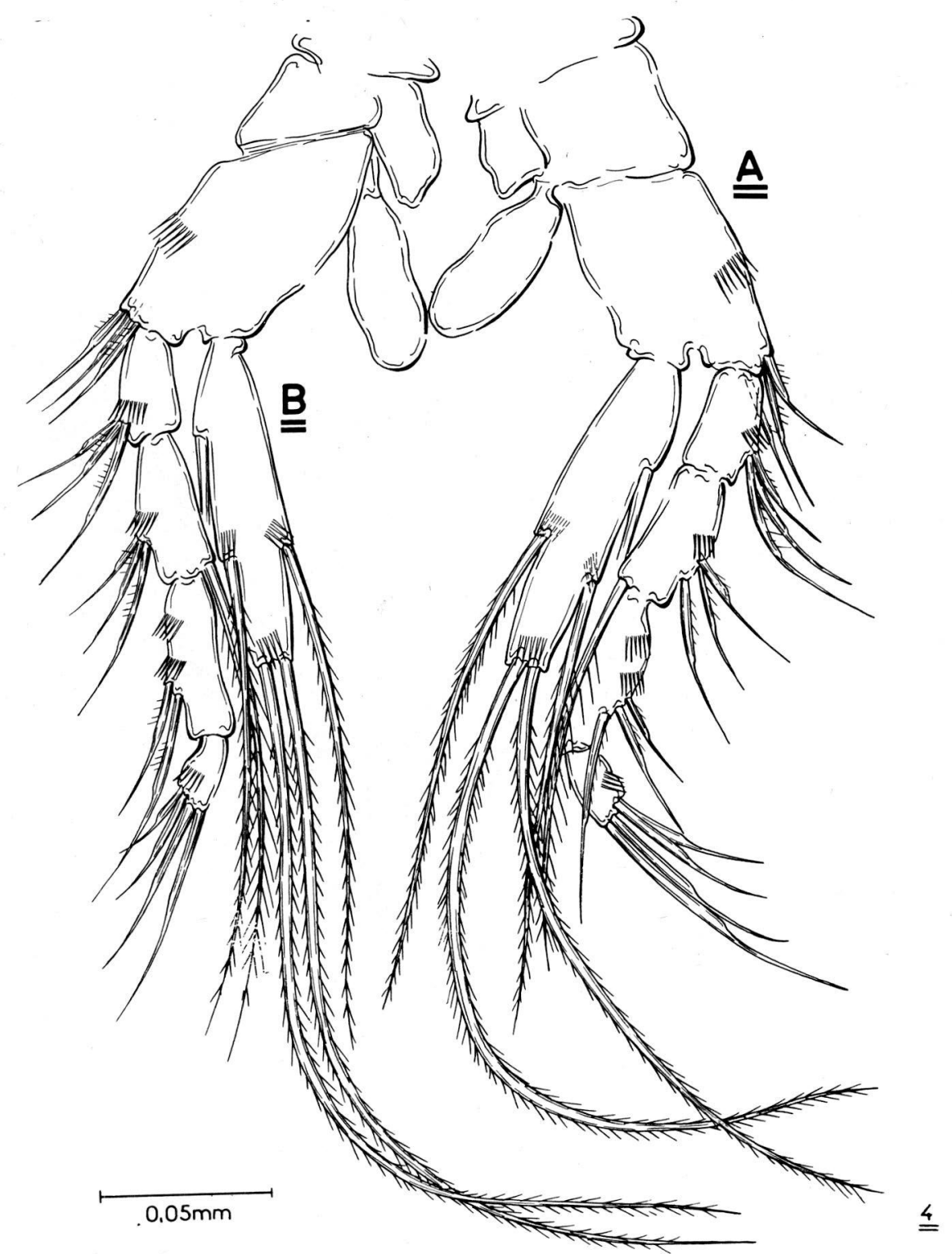

Planche 4. Bathynella (Bathynella) boteai Serban. A, péréiopode III; B, péréiopode IV. 


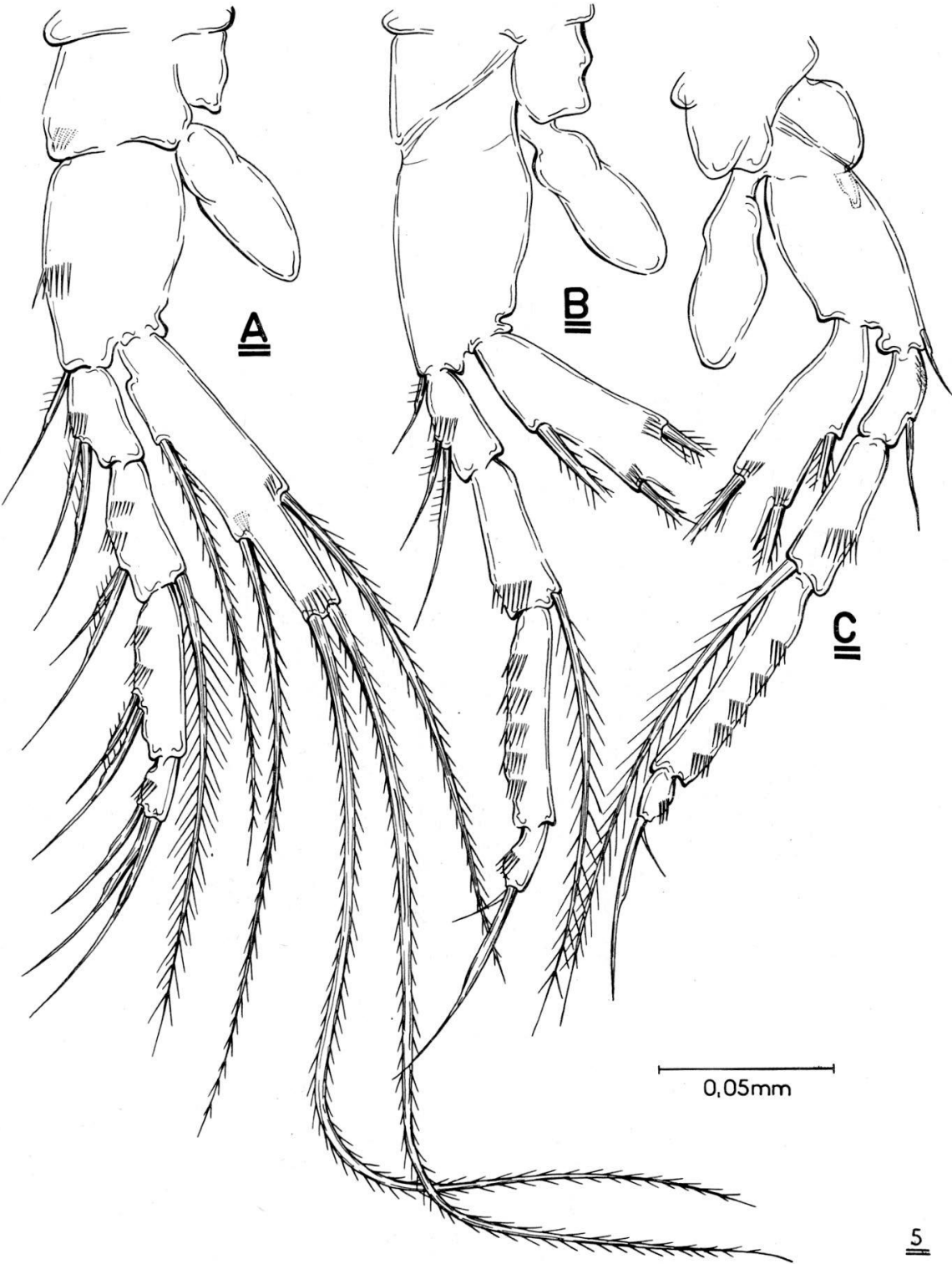

Planche 5. (Bathynella (Bathynella) boteai Serban. A, péréiopode V; B, péréiopode VI; C, péréiopode VII. 
que les péréiopodes IV. Selon nous, cette chétotaxie particulière des péréiopodes $\mathrm{V}$ de $B$.(B.) boteai représente l'élément le plus important qui détermine sur les appendices VI et VII une chétotaxie semblables à celle de $B .(B$.) paranatans, le décalage numérique des quatre premières paires entre les deux espèces étant réduit considérablement chez la cinquième (tableau 1-4). Notons encore deux faits: les péréiopodes $\mathrm{V}$ de $B$. $(B$.) boteai ont une chétotaxie qui perd tous les traits essentiels caractérisant les paires précédentes, ou plus précisement, la chétotaxie des appendices ambulatoires de $B$.(B.) boteai: on ne trouve, ni les 4 poils apicaux sur l'endopodite, ni un nombre élevé de phanères sur le basipodite ( 3 ou 5 polis). La combinaison numérique de la chétotaxie des péréiopodes $\mathrm{V}$ de $B$.(B.) boteai est pour les mâles et respectivement pour les femelles la même que celle des péréiopodes IV ( $\left.\delta^{\circ}\right), \mathrm{V}(\%)$ de B.(B.) paranatans.

f) il est intéressant de noter que le nombre plus élevé de poils présents chez $B .(B$.$) boteai dans les deux premières catégories de péréiopodes se trouve dans une$ certaine mesure même sur les paires VI et VII; il ne s'agit pas seulement de l'exopodite des péréiopodes VII, portant 5 poils, mais aussi des 2 poils de l'article endopodial I des péréiopodes VI; c'est pour la première fois que nous avons rencontré ce dernier trait chez une espèce de Bathynella;

g) les articles III de l'endopodite des péréiopodes VI sont munis de 4-5 cténidies;

h) enfin, il faut mentionner que chez une femelle les péréiopodes IV portent 3 poils apicaux, tandis que chez un mâle, l'un de ces appendices en a 3 et l'autre 4 (il s'agit de l'endopodite).

Péréiopode VIII mâle (Pl. 6, 7, 8A). Dans la contribution comprenant la description initiale de l'espèce (Serban, 1971), nous avons accordé une attention particulière à la partie apicale de la plaque antérieure dont la forme est caractéristique des 4 représentants de Roumanie. Dans une note plus récente (Serban, 1973b) il a été souligné que la plaque antérieure, vue du côté latéro-externe, présente 2 petites proéminences apicaleș chez B.(B.) paranatans, 2 mamelons chez B.(B.) motren$s i s$, un prolongement chez $B .(B$.) boteai et deux prolongements pointus chez B.(B.) plesai. L'étude des autres représentants du genre (voir B.(B.) vaducrisensis n. sp.) nous a obligé de pousser plus loin l'analyse des péréiopodes VIII mâles, car la forme de la partie apicale de la plaque antérieure, à elle seule, ne suffit pas toujours à l'identification d'une espèce; il y a des cas où, en dépit de la différenciation accentuée d'une longue série de caractères, la plaque antérieure possède une structure identique à celle d'autres espèces. C'est cette raison qui nous a déterminé à chercher d'autres traits différentiels et, comme on le verra, l'un des plus significatifs semble être la forme du bord de la proéminence externe de la région basale du complexe pénien.

Vue de face (Pl. 6A), la plaque antérieure du pénis de B.(B.) boteai (Plq-ant) présente deux parties bien individualisées: la moitié basale, bien développée en largeur et la moitié distale, nettement plus mince et à diamètre constant sur la majeure partie de sa longueur. L'existence de ces deux parties est due surtout à la forme du bord externe de la plaque qui, étant légèrement incliné vers le plan sagittal du pénis dans sa partie basale, change de direction en descendant verticalement dans sa région apicale. Chez les autres espèces, ce bord est soit convexe (B./B.) paranatans et B.(B.) motrensis), soit droit (B.(B.) plesai). 


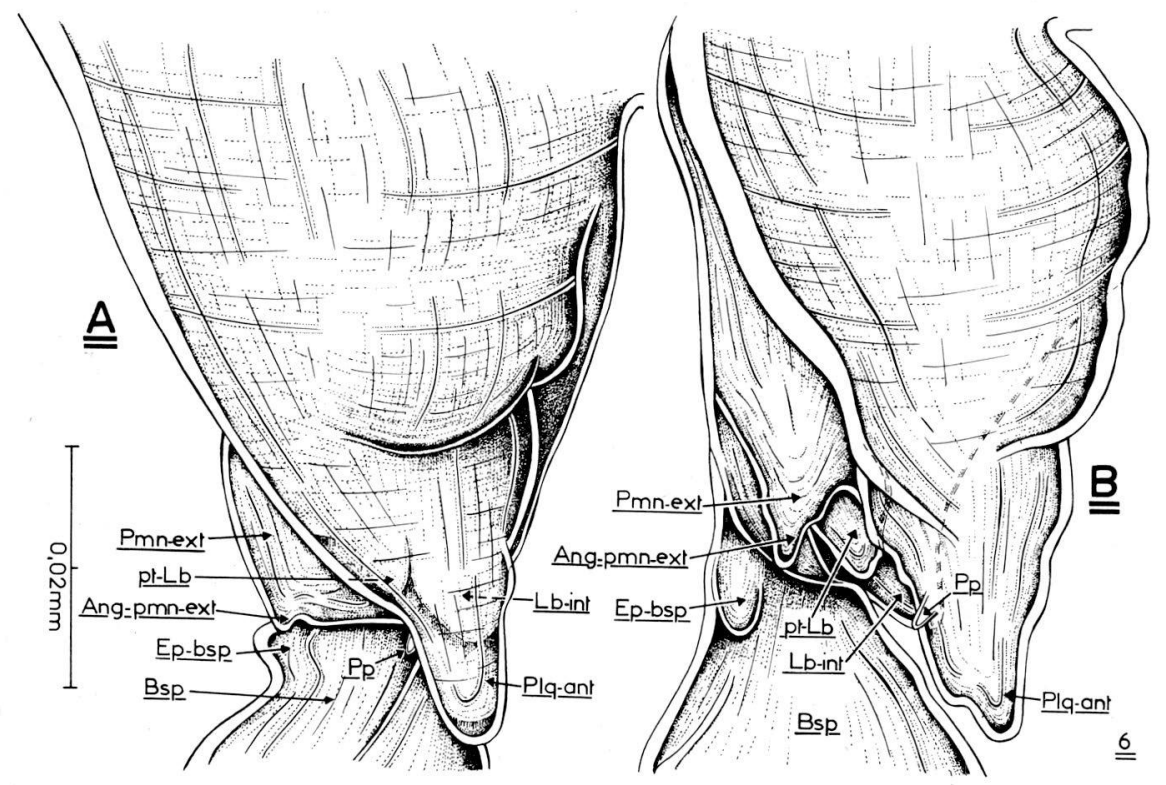

Planche 6. Bathynella (Bathynella) boteai Serban. Partie pénienne du péréiopode VIII mâles: A, face rostrale; B, face latéro-externe Ang-pmn-ext, angle de la proéminence externe; Bsp, basipodite; Ep-bsp, éperon du basipodite; Lb-int, lobe interne; Plq-ant, plaque antérieure; Pm-ext, proéminence externe; $\mathrm{Pp}$, papille; pt-Lb, petit lobe.

Sur la face latéro-externe du pénis, la plaque antérieure prend l'aspect d'un lobe conique dont la partie terminale porte, de son côté rostral, un prolongement bien développé. La papille ( $\mathrm{Pp})$ est aisément visible.

En dessous de la plaque il y a le lobe interne (Lb-int) et le petit lobe (pt-Lb); le premier, moins robuste que la plaque antérieure, occupe la partie médiale de l'appendice; le second, à position externe par rapport au lobe interne, est une formation bien plus petite dont la largeur apicale est deux fois plus courte que sa longueur.

Enfin, un caractère non mentionné jusqu'à présent, est la forme rostrale ou caudale de la proéminence externe de la région basale du complexe pénien. Comme on le voit dans les planches $6 \mathrm{~A}$ et $7 \mathrm{~A}$, la proéminence externe (Pmn-ext) a les bords externe et ventral perpendiculaires, formant un angle que nous dénommons l'angle de la proéminence externe (Ang-pmn-ext.)

Péréiopode VIII femelle (Pl. 8B). L'exopodite, de longueur relativement égale à celles du basipodite et de l'endopodite prises ensemble, porte 4 phanères dans sa région terminale. Epipodite respiratoire de grande taille.

Pléopode (PL. 8C). Article distal à 4 poils.

Uropode (Pl. 8 E,F). Sur le sympodite on rencontre 7-8 épines. L'endopodite porte, outre les 4 poils généralement présents chez les espèces du genre Bathynella, 


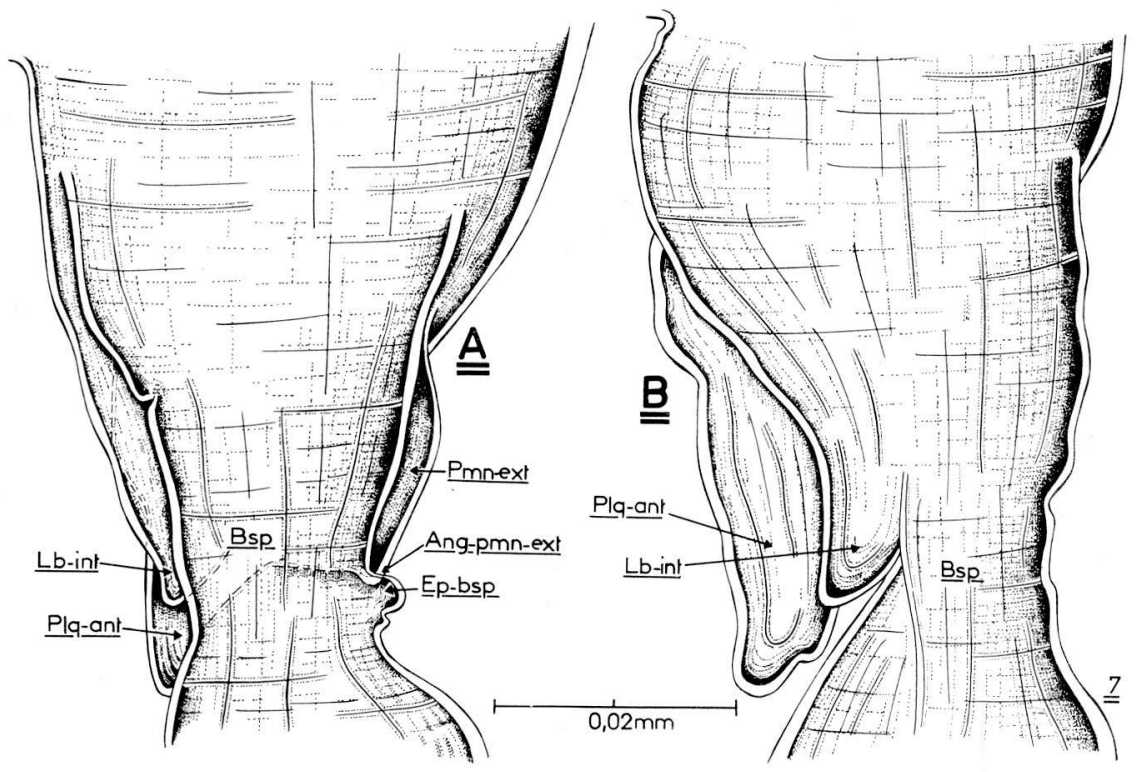

Planche 7. Bathynella (Bathynella) boteai Serban. Partie pénienne du péréiopode VIII mâle: A, face caudale; B, face latéro-interne; Ang-pmn-ext, angle de la proéminence externe; Bsp, basipodite; Ep-bsp, éperon du basipodite; Lb-int, lobe interne; Plq-ant, plaque antérireure; Pmn-ext, proéminence externe.

4 griffes; la quatrième, ayant une longueur semblable à celle de l'endopodite, est respectivement 2 fois, 2,5 et 3,5 à 4 fois plus longue que la troisième, la deuxième et la première griffe.

Furca (P1. 8D). Le poil 1 est, généralement, deux fois plus long que le bras furcal; les poils 2 et 3 , de taille semblable, atteignent les $4 / 5$ de la longueur du premier; le poil 4 est d'un tiers plus court que les deux précédents; le poil dorsal, de taille réduite, est respectivement environ deux et trois fois plus court que les poils 4 et 1 .

Diagnose. Péréiopode VIII mâle à plaque antérieure terminée par un prolongement rostral; proéminence externe à bord en angle droit. Chétotaxie des péréiopodes ambulatoires composée d'un riche nombre de poils; première paire à 54 phanères; le quatrième article endopodial des péréiopodes I-IV à 4 poils; combinaison numérique fondamentale de la chétotaxie $=0 / 03 / 33 / 33 / 33 / 34 / 45 / 5$; exopodite des péréiopodes VII à 5 poils. Article distal du pléopode muni de 4 phanères. Uropode à 4 griffes endopodiales et 7-8 épines sur 1e sympodite. Poils furcaux 2 et 3 , de taille semblable, plus courts que le poil 1; poil dorsal, deux fois plus court que le poil 4. Antennule sans poil interne et proximal sur l'article I du pédoncule. Article endopodial II de l'antenne, de la même longueur que l'article V; poil médian de l'exopodite, absent. 


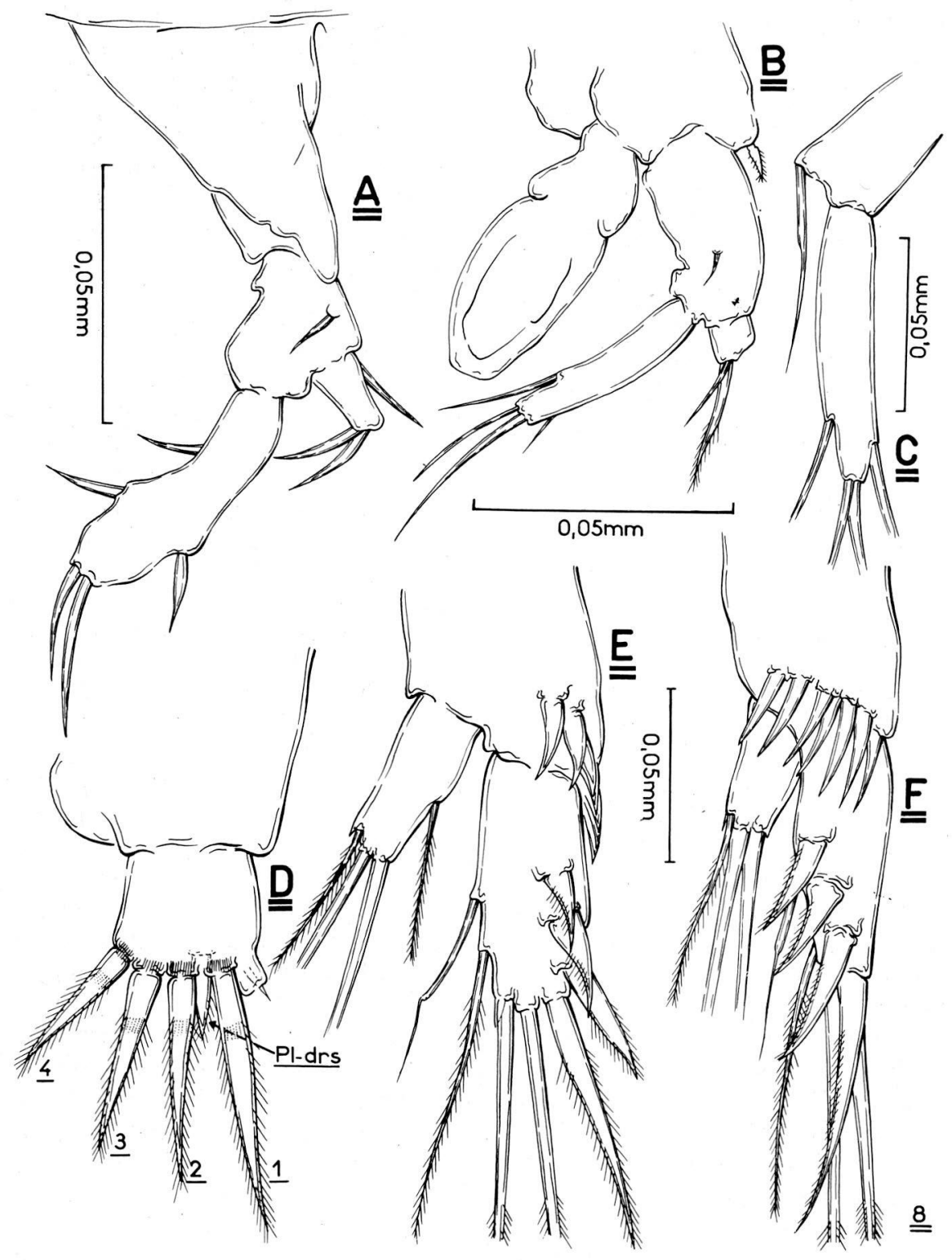

Planche 8. Bathynella (Bathynella) boteai Serban. Péréiopode VIII mäie, vue générale; B, péréiopode VIII femelle; C, pléopode; $D$, furca, vue ventrale; E,F, uropode. 1,2,3,4, les poils apicaux de la furca; Pl-drs, poil dorsal. 
Remarques

Dans la première description de l'espèce (Serban, 1971), nous avons précisé que, selon les données concernant les Bathynella d'Europe, B.(B.) boteai peut être synonyme de $B$. chappuisi Delachaux. D'ailleurs, cette possibilité n'était pas à exclure: d'une part, l'un des caractères les plus saillants de notre espèce, les 4 griffes de l'endopodite des uropodes, se rencontre aussi chez $B$. chappuisi; d'autre part, la description très sommaire de cette dernière espèce a rendu impossible la mise en évidence des traits séparant les deux taxa. A ces deux faits vient s'ajouter un autre:

Tableau 5. Bathynella (Bathynella) boteai Serban et

Bathynella chappuisi Delachaux,

caractẽres differentiels

\begin{tabular}{|c|c|c|}
\hline Caractère & $\frac{\text { B. (B.) boteai }}{\text { Serban }}$ & $\frac{\text { B. chappuisi }}{\text { Delachaux }}$ \\
\hline & - 4 dents à épines & - 3 dents à épines \\
\hline $\begin{array}{l}\text { péréiopodes } \\
\text { I-VII }\end{array}$ & $\begin{array}{l}\text { - } \mathrm{P}_{\mathrm{I}} \text { sans épipodite } \\
\text { respiratoire } \\
\text { - article endopodial IV } \\
\text { des } \mathrm{P}_{\mathrm{I}} \mathrm{P}_{\mathrm{IV}} \text { à } 4 \text { phanères } \\
\text { - combinaison numérique } \\
\text { fondamentale: } \\
0 / 03 / 33 / 33 / 33 / 34 / 45 / 5\end{array}$ & $\begin{array}{l}\text { - }{ }{ }_{I} \text { à épipodite } \\
\text { respiratoire } \\
\text { - article endopodial IV } \\
\text { des } P_{I}-P_{V} \text { à } 3 \text { phanères } \\
\text { - combinaison numérique } \\
\text { fondamentale: } \\
0 / 02 / 22 / 22 / 22 / 23 / 35 / 5\end{array}$ \\
\hline $\begin{array}{l}\text { péréiopode } \\
\text { VIII mâle }\end{array}$ & $\begin{array}{l}\text { - plaque antérieure avec } \\
\text { un prolongement termi-- } \\
\text { nal et rostral } \\
\text { - proéminence externe à } \\
\text { contour en angle droit } \\
\text { - complexe pénien formé } \\
\text { de } 3 \text { lobes }\end{array}$ & $\begin{array}{l}\text { - plaque antérieure avec } \\
\text { deux mamelons terminaux } \\
\text { - proéminence externe } \\
\text { semi-circulaire } \\
\text { - complexe pénien forme } \\
\text { de } 4 \text { lobes }\end{array}$ \\
\hline pléopode & $\begin{array}{l}\text { - à } 4 \text { poils sur l'article } \\
\text { distal }\end{array}$ & $\begin{array}{l}\text { - à } 5 \text { poils sur l'article } \\
\text { distal }\end{array}$ \\
\hline uropode & - sympodite à 7-8 épines & - sympodite à 6 épines \\
\hline furca & $\begin{array}{l}\text { - poils } 2 \text { et } 3 \text { de la même } \\
\text { taille } \\
\text { - poil dorsal beaucoup } \\
\text { plus court que le poil } 4\end{array}$ & $\begin{array}{l}\text { - poils } 1 \text { et } 2 \text { de la même } \\
\text { taille } \\
\text { - poil dorsal moins court } \\
\text { que le poil } 4\end{array}$ \\
\hline
\end{tabular}


P.A. Chappuis $(1924-1925$, 1944) et P. Bartok (1944) ont soutenu que B. chappuisi est largement répandu dans les eaux souterraines de Transylvanie. Tout récemment, grâce à l'amabilité de M.J.G. Bear de l'Université de Neuchâtel et de M.V. Aellen du Muséum de Genève, E. Serban et Cl. Delamare Deboutteville (1974) ont pu étudier le matériel original de B. chappuisi. Si l'on compare maintenant $B$. (B.) boteai à $B$. chappuisi, on voit qu'en dehors de leur ressemblance au niveau de la chétotaxie de l'endopodite des uropodes, les autres caractères sont bien différents (tableau 5). Rappelons également que la probabilité de l'existence d'un lobe parapénien dans la morphologie du péréiopode VIII mâle de $B$. chappuisi sépare cette espèce de tous les représentants du sous-genre Bathynella (Bathynella) tout en la rapprochant de B.(L.) lombardica, espèce type du sous-genre Bathynella (Lombardobathynella) Serban, 1973 a).

Compte tenu des Bathynella d'Europe dont la description est plus détaillée, $B$.(B.) boteai s'individualise, d'après la chétotaxie des péréiopodes, comme une forme tout à fait particulière. Même si l'on considère certains représentants de la sous famille des Gallobathynellinae Serban, Coineau et Delamare Deboutteville ayant l'article apical de certaines paires de péréiopodes muni de 4 poils, le nombre de 54 phanères sur les péréiopodes I ainsi que la combinaison numérique fondamentale dont l'élément le plus fréquent est le nombre de 3 poils restent des traits tout à fait spécifiques de $B$.(B.) boteai.

\section{Bathynella (Bathynella) vaducrisensis n.sp. (Pl. 9-15)}

Matériel. Plusieurs exemplaires collectés par C. Plesa du milieu interstitiel de la rivière souterraine de la grotte „Pestera Vadul Crisului” (,Pestera lui Zichy”), dép. de Bihor, Transylvanie. La capture systématique de la faune souterraine aquatique de cette grotte (1957-1961) a permis à notre collègue de trouver plus de $2000 \mathrm{Ba}$ thynella (Plesa, 1967) appartenant à plusieurs espèces; l'espèce $B$. (B.) vaducrisensis n.sp. semble être peu représentée en comparaison avec les autres; l'étude de ces dernières fera l'objet d'une autre contribution. Quelques observations sur la morphologie de B.(B.) vaducrisensis n.sp. ont été insérées dans notre étude sur Bathynella (Serban, 1972); comme il a déjà été mentionné, Bathynella sp, dénomination provisoire utilisée alors, n'est en fait que B.(B.) vaducrisensis dont les sclérites cuticulaires ont été décrits (Serban, 1972, Pl.LXI).

Taille: $1 \mathrm{~mm}-1,2 \mathrm{~mm}$.

Antennule (P1.9 A). L'article basal du pédoncule n'a qu'un seul poil sur sa face interne.

Antenne (Pl.9 B). L'article endopodial II, plus court que le cinquième, est de la même longueur que l'article IV. Le poil médian n'est pas développé sur l'exopodite; ramification apicale de l'organe sensoriel similaire à celle de $B$. $(B$.) boteai.

Mandibule (Pl.9 C). Partie masticatrice à structure typique. La dernière dent porte sur son bord un petit denticule.

Maxillule (fig.9 D). Toutes les dents maxillulaires munies de 2 épines; sur l'une des dents apicales, elles sont très réduites.

Maxille (pl.9 E). Les 5 phanères du palpe sont des poils endopodiaux simples. 


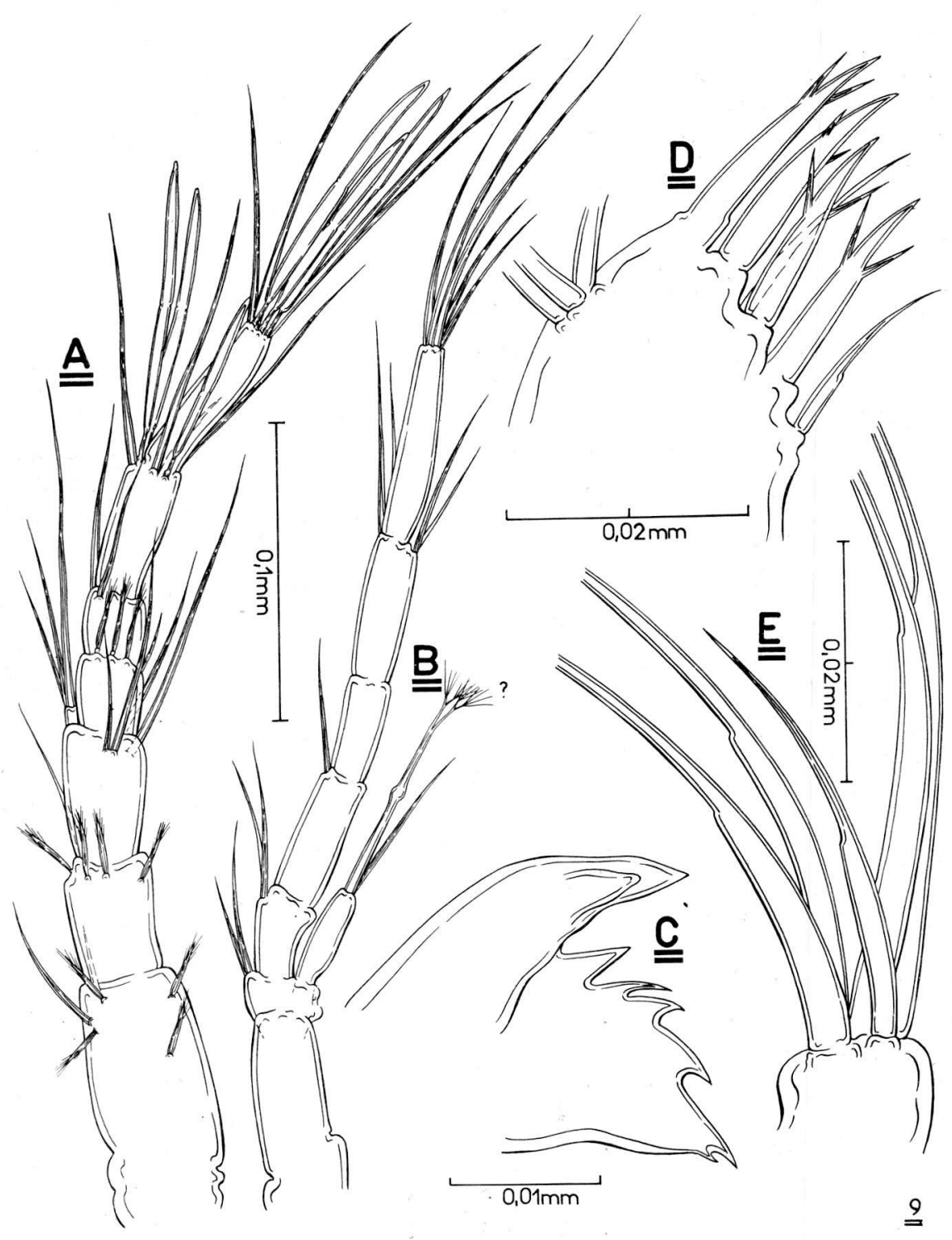

Planche 9. Bathynella (Bathynella) vaducrisensis n.sp. A, antennule; B, antenne; C, partie masticatrice de la mandibule; $\mathrm{D}$, région distale de la maxillule; $\mathrm{E}$, les poils du palpe maxillaire. 
Péréiopodes I-VII (P1. 10-12). L'épipodite respiratoire n'est pas développé sur les péréiopodes I. Vu de face, l'exite précoxal diffère de celui de B.(B.) boteai, ayant le bord arrondi. Eperon coxal des péréiopodes VII mâles, court. Quant à la chétotaxie (tableaux 6 et 7), il y a lieu de faire les observations suivantes:

a) considérée dans son ensemble, la chétotaxie des péréiopodes rapproche l'espèce en question de $B$.(B.) boteai, le nombre total des poils, leur répartition sur les diverses paires et l'identité de la combinaison numérique fondamentale prouvant des affinités incontestables; toutefois, le nombre maximal et le nombre minimal de poils, tous le deux plus réduits, la fréquence -elle aussi plus réduite- des 4 poils

Tableau 6. Bathynella (Bathynella) vaducrisensiłs n.sp. Combinaisons numériques de la chétotaxie des péréiopodes I-VII (mâle)

\begin{tabular}{lcccccccc}
\hline Péréiopode & Cx & Bsp & I & II & III & IV & Exp & $\begin{array}{c}\text { Nombre total } \\
\text { de poils }\end{array}$ \\
\hline I & $1 / 1$ & $3 / 3$ & $5 / 5$ & $3 / 3$ & $3 / 3$ & $4 / 4$ & $5 / 5$ & 48 \\
II & $0 / 0$ & $3 / 3$ & $3 / 3$ & $3 / 3$ & $3 / 3$ & $4 / 4$ & $5 / 5$ & 42 \\
III & $0 / 0$ & $3 / 3$ & $3 / 3$ & $3 / 3$ & $3 / 3$ & $4 / 4$ & $5 / 5$ & 42 \\
IV & $0 / 0$ & $2 / 2$ & $3 / 3$ & $3 / 3$ & $3 / 3$ & $3 / 3$ & $5 / 5$ & 38 \\
V & $0 / 0$ & $1 / 1$ & $2 / 2$ & $2 / 2$ & $2 / 2$ & $3 / 3$ & $5 / 5$ & 30 \\
VI & $0 / 0$ & $1 / 1$ & $1 / 1$ & $0 / 0$ & $0 / 0$ & $2 / 2$ & $5 / 5$ & 18 \\
VII & $0 / 0$ & $1 / 1$ & $1 / 1$ & $0 / 0$ & $0 / 0$ & $2 / 2$ & $4 / 4$ & 16
\end{tabular}

Tableau 7. Bathynella (Bathynella) vaducrisensis n.sp. Combinaisons numériques de la chétotaxie des péréiopodes I-VII (femelle)

\begin{tabular}{lcccccccc}
\hline Péréiopode & Cx & Bsp & I & II & III & IV & Exp & $\begin{array}{c}\text { Nombre total } \\
\text { de poils }\end{array}$ \\
\hline I & $1 / 1$ & $3 / 3$ & $5 / 5$ & $4 / 4$ & $3 / 3$ & $4 / 4$ & $5 / 5$ & 50 \\
II & $0 / 0$ & $3 / 3$ & $3 / 3$ & $3 / 3$ & $3 / 3$ & $4 / 4$ & $5 / 5$ & 42 \\
III & $0 / 0$ & $3 / 3$ & $3 / 3$ & $3 / 3$ & $3 / 3$ & $4 / 4$ & $5 / 5$ & 42 \\
IV & $0 / 0$ & $2 / 2$ & $3 / 3$ & $3 / 3$ & $3 / 3$ & $3 / 3$ & $5 / 5$ & 38 \\
V & $0 / 0$ & $1 / 1$ & $2 / 2$ & $2 / 2$ & $2 / 2$ & $3 / 3$ & $5 / 5$ & 30 \\
VI & $0 / 0$ & $1 / 1$ & $1 / 1$ & $0 / 0$ & $0 / 0$ & $2 / 2$ & $5 / 5$ & 18 \\
VII & $0 / 0$ & $1 / 1$ & $1 / 1$ & $0 / 0$ & $0 / 0$ & $2 / 2$ & $4 / 4$ & 16
\end{tabular}


sur l'article endopodial IV des divers péréiopodes, ainssi que le nombre des phanères surl'exopodite des appendices VII sont des éléments qui individualisent $B .(B$. vaducrisensis n.sp. par rapport à $B$.(B.) boteai;

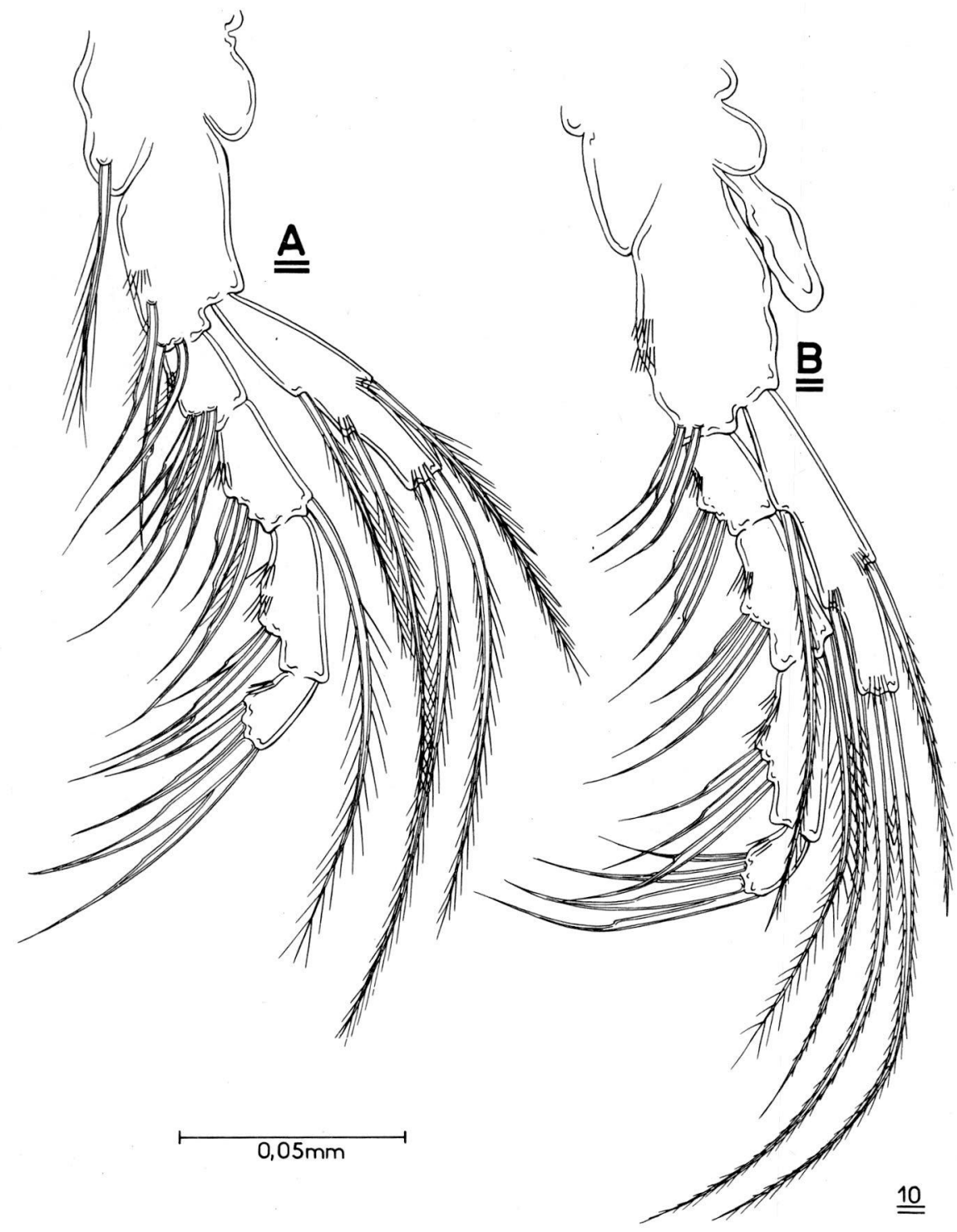

Planche 10. Bathynella (Bathynella) vaducrisensis n.sp. A, péréiopode I, B, péréiopode II. 


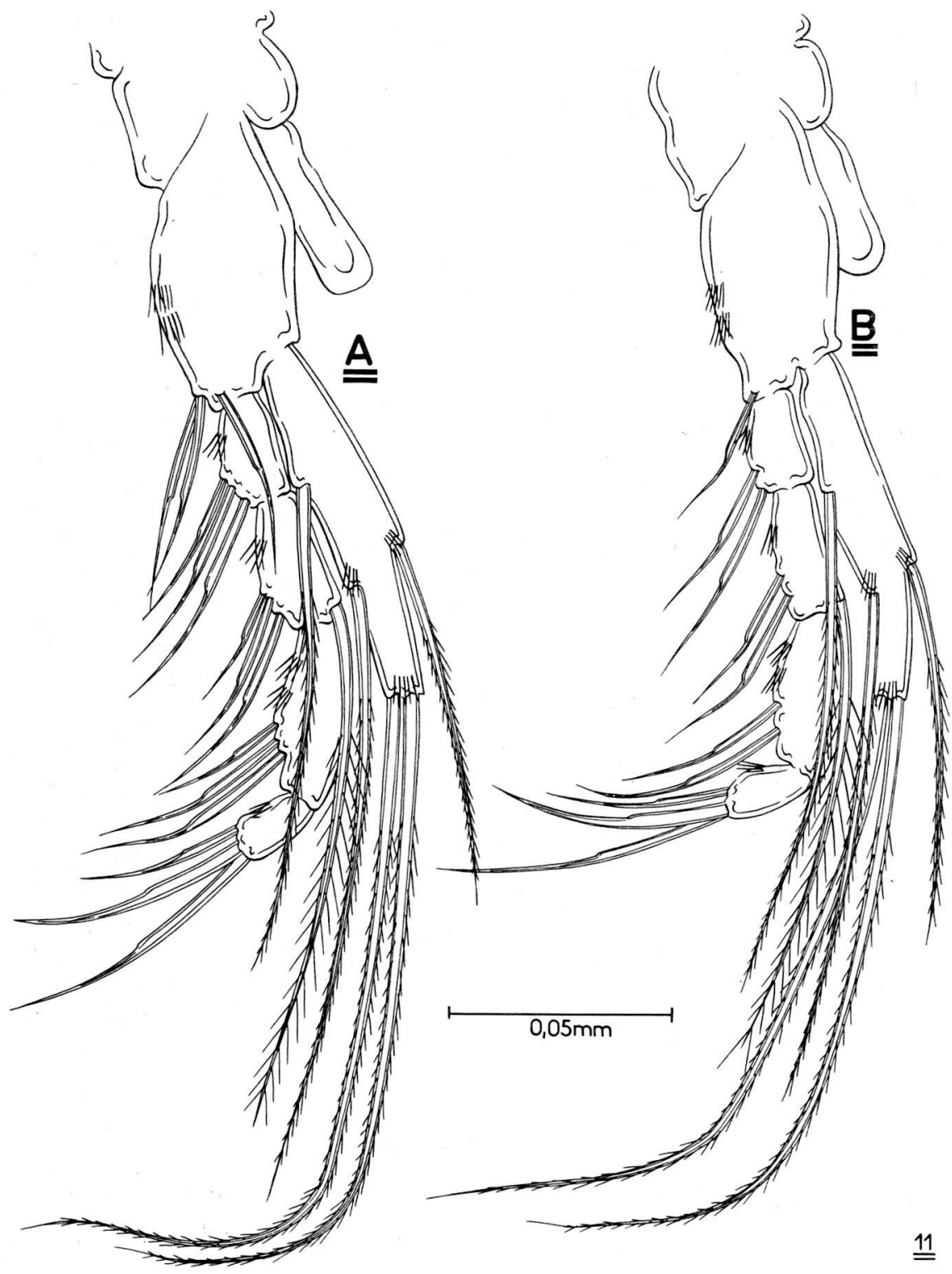

Planche 11. Bathynella (Bathynella) vaducrisensis n.sp. A, péréiopode III; B, péréiopode V. 


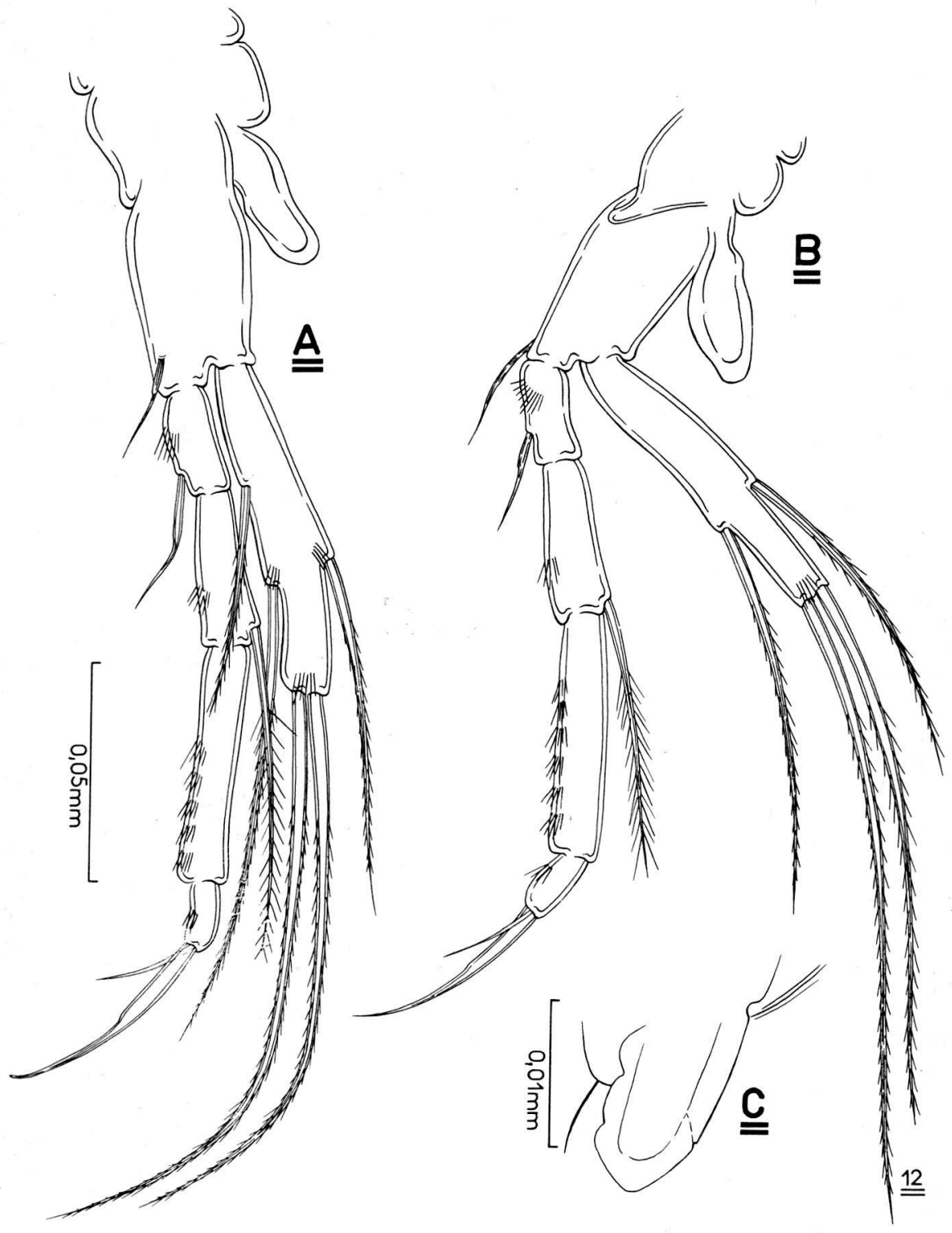

Planche 12. Bathynella (Bathynella) vaducrisensis n.sp. A, péréiopode VI; B, péréiopode VII; C, éperon coxal du péréiopode VII mâle. 


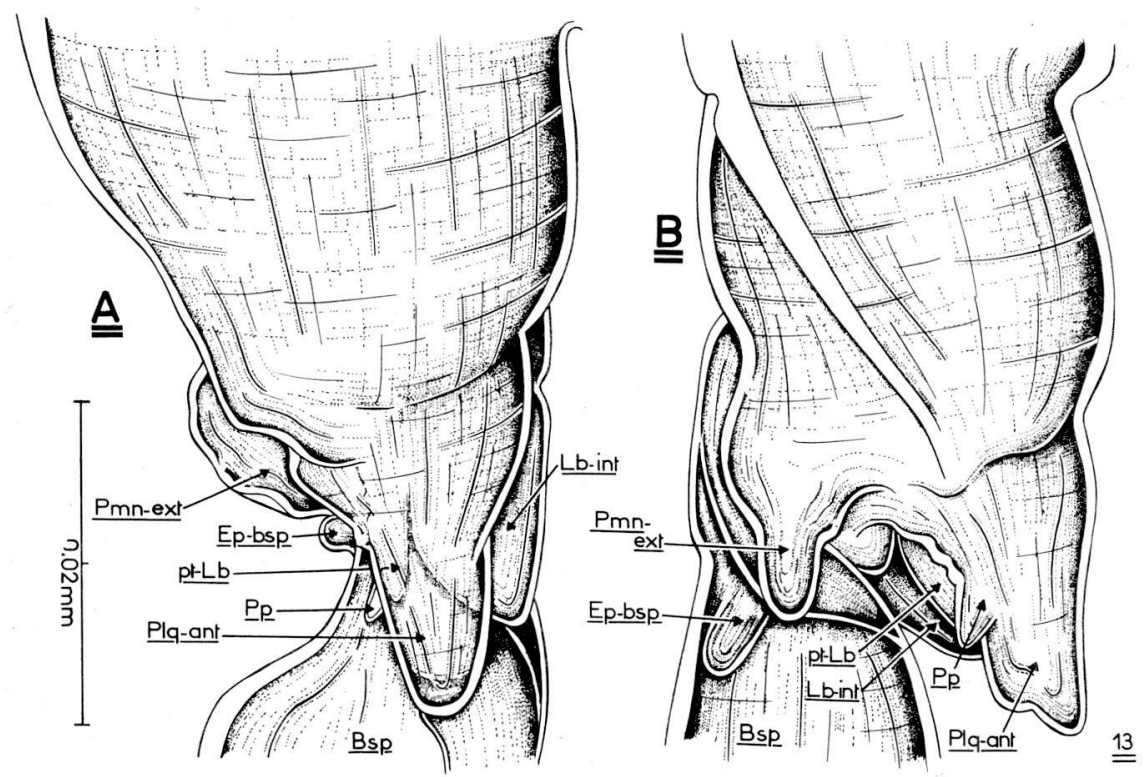

Planche 13. Bathynella (Bathynella) vaducrisensis n.sp. Partie pénienne du péréiopode VIII mâle: A, face rostrale; B, face latéro-externe. Bsp, basipodite; Ep-bsp, éperon du basipodite; Lb-int, lobe interne; Plq-ant, plaque antérieure; Pmn-ext, proéminence externe; Pp, papille; pt-Lb, petit lobe.

b) chez B.(B.) vaducrisensis n.sp., le basipodite ne porte jamais plus de 3 poils, les péréiopodes IV n'ont jamais 4 poils sur l'article terminal de l'endopodite et la combinaison numérique fondamentale de la chétotaxie ne se rencontre que sur les paires II et III.

Péréiopode VIII mâle (Pl. 13, 14). La plaque antérieure (plq-ant) est pareille à celle de $B$.(B.) boteai. La proéminence externe de la région basale du complexe pénien (Pmn-ext) montre une structure caractéristique, son bord externe étant arrondi (Pl. 13A, 14A). Un autre trait qui distingue l'espèce, c'est le petit lobe (pt-Lb), plus allongé que celui de B.(B.) boteai. Comme on peut le voir sur la figure 13, la partie terminale du lobe se trouve au même niveau que la papille de la plaque antérieure, situation qui ne se rencontre pas chez B.(B.) boteai où le lobe est visiblement plus court (Pl. 6).

Péréiopode VIII femelle (Pl. 15A). Les 2 poils situés sur les bords de l'exopodite se trouvent à la limite distale du tiers médian de la rame; chez B.(B.) boteai, ils sont plus proches de l'extrémité apicale.

Pléopode ( $\mathrm{Pl}$. 15B). L'article distal est muni de 3 poils.

Uropode (Pl. 15C). Le sympodite porte 8-9 épines; le nombre maximal est plus fréquent chez les femelles que chez les mâles. Les 3 griffes de l'endopodite ont les dimensions suivantes: la griffe distale est de la même longueur que la rame; 

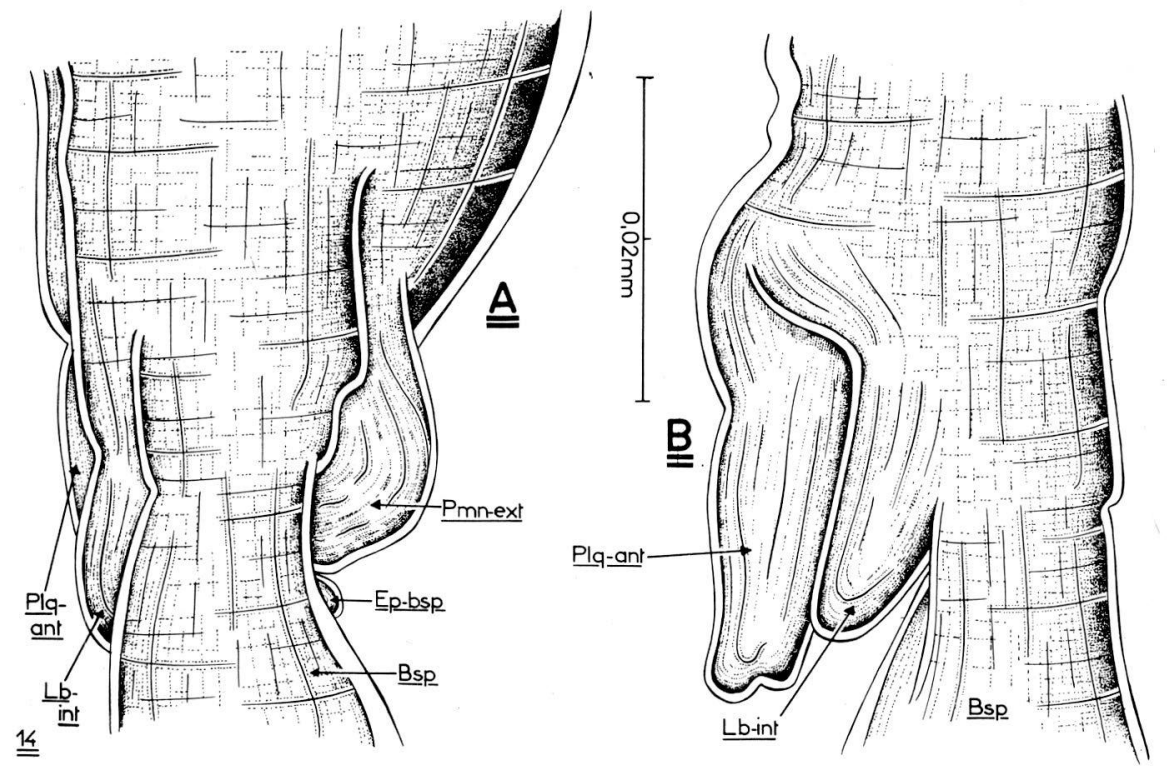

Planche 14. Bathynella (Bathynella) vaducrisensis n.sp. Partie pénienne du péréiopode VIII mâle: A, face caudale; B, face latéro-interne. Bsp, basipodite; Ep-bsp, éperon du basipodite; Lb-int, lobe interne; Plq-ant, plaque antérieure; Pmn-ext, proéminence externe.

la griffe médiane atteint les $2 / 3$ de la précédente; la griffe proximale égale un tiers de la longueur de la griffe distale.

Furca (P1. 15D,E). Le poil 1, qui dépasse d'un tiers de sa longueur le bras furcal, est égal au poil 2; le poil 3, atteignant deux tiers de la longueur des deux précédents, est presque deux fois plus long que le poil 4; le poil dorsal, environ trois fois plus court que le poil 1 , égale le quatrième.

Diagnose. Péréiopode VIII mâle à plaque antérieure semblable à celle de $B .(B$.) boteai; proéminence externe à bord arrondi. Péréiopodes I portant 48 -50 phanères; paires I-III des deux sexes à 4 poils sur l'article endopodial IV; combinaison numérique fondamentale de la chétotaxie $=0 / 03 / 33 / 33 / 33 / 34 / 45 / 5$; exopodite des péréiopodes VII muni de 4 poils. Pléopode à 3 poils sur l'article distal. Endopodite des uropodes à 3 griffes; sympodite portant 8 -9 épines. Poils furcaux 1 et 2 de taille semblable; poil dorsal et poil 4 de la même longueur. Antennule comme chez $B$.(B.) boteai. Antenne ayant l'article endopodial II plus court que le cinquième; poil médian de l'exopodite, absent.

\section{Remarques}

La manière par laquelle s'individualise $B$.(B.) vaducrisensis par rapport à $B .(B$.) boteai, espèces dont la proche parenté est incontestable, révèle un nouvel aspect 


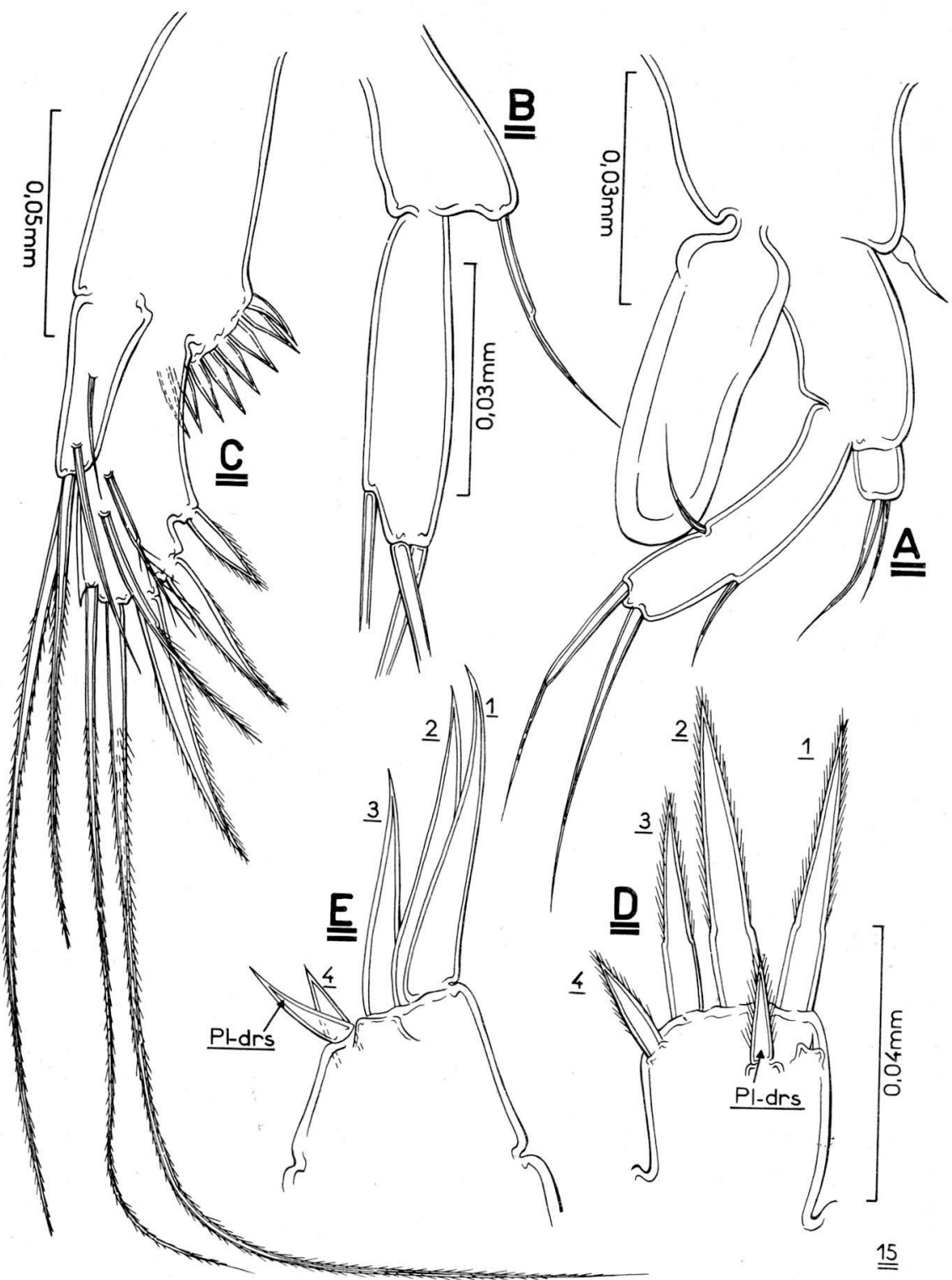

Planche 15. Bathynella (Bathynella) vaducrisensis n.sp. A, péréiopode VII femelle; B, pléopode; C, uropode; D, furca, vue dorsale; E, furca, vue latéro-externe. 1,2,3,4, poils apicaux de la furca; Pl-drs, poil dorsal. 
Tableau 8. Bathynella (Bathynella) boteai Serban et Bathynella (Bathynella) vaducrisensis n.sp., similitudes et différences

\begin{tabular}{|c|c|c|}
\hline Caractère & $\frac{\text { B. (B.) boteai }}{\text { Serban }}$ & $\frac{\text { B.(B.) vaducrisensis }}{\text { n.sp. }}$ \\
\hline antennule & $\begin{array}{l}\text { - poil interne et ba } \\
\text { du pédoncule non d }\end{array}$ & $\begin{array}{l}\text { al de l'article I } \\
\text { veloppe }\end{array}$ \\
\hline antenne & $\begin{array}{l}\text { - poil médian de I'ex } \\
\text { - articles endopodiaux II } \\
\text { et V de la même longueur }\end{array}$ & $\begin{array}{l}\text { opodite, absent } \\
\text { - article endopodial II plus } \\
\text { court que l'article V }\end{array}$ \\
\hline maxillule & - 4 dents à épines & \\
\hline maxille & $\begin{array}{l}\text { - palpe à } 3 \text { poils endopo- } \\
\text { diaux clliés sur le bord } \\
\text { externe et } 2 \text { poils sim- } \\
\text { ples }\end{array}$ & - palpe à 5 poils simples \\
\hline $\begin{array}{l}\text { péréiopodes } \\
\text { I-VII }\end{array}$ & 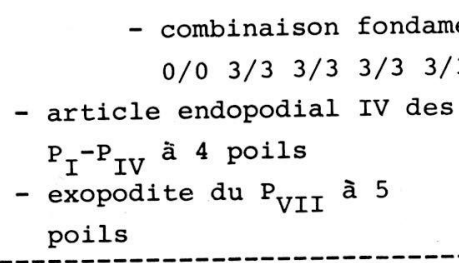 & $\begin{array}{l}\text { ntale de la chétotaxie: } \\
4 / 45 / 5 \\
\text { - article endopodial IV des } \\
\text { PI }^{-P} \text { III à } 4 \text { poils } \\
\text { - exopodite du P }{ }_{\text {VII }} \text { à } 4 \\
\text { poils }\end{array}$ \\
\hline $\begin{array}{l}\text { péréiopode } \\
\text { VIII mâle }\end{array}$ & $\begin{array}{l}\text { - région terminale d } \\
\text { avec un prolongeme } \\
\text { - proéminence externe à } \\
\text { contour rectangulaire }\end{array}$ & $\begin{array}{l}\text { la plaque antérieure } \\
\text { tostral } \\
\text { - proéminence externe à } \\
\text { contour semi-circulaire }\end{array}$ \\
\hline pléopode & - article distal à 4 poils & - article distal à 3 poils \\
\hline uropode & $\begin{array}{l}\text { - sympodite à } 7-8 \text { épines } \\
\text { - endopodite à } 4 \text { griffes }\end{array}$ & $\begin{array}{l}\text { - sympodite à 8-9 épines } \\
\text { - endopodite à } 3 \text { griffes }\end{array}$ \\
\hline furca & $\begin{array}{l}\text { - poils } 2 \text { et } 3 \text { de taille } \\
\text { semblable } \\
\text { - poil dorsal } 2 \text { fois plus } \\
\text { court que le poil } 4\end{array}$ & $\begin{array}{l}\text { - poils } 1 \text { et } 2 \text { de taille } \\
\text { semblable } \\
\text { - poil dorsal de la même } \\
\text { longueur que le poil } 4\end{array}$ \\
\hline
\end{tabular}


du processus de la spéciation dans le sous-genre Bathynella (Bathynella). On constate des remaniements au niveau de plusieurs appendices qui, tout en étant similaires par certains traits, sont, en même temps, bien différenciès selon d'autres. Même les éléments qui prouvent l'affinité des deux espèces, à savoir la chétotaxie des péréiopodes I-VII et la morphologie des péréiopodes VIII mâles, présentent, à côté d'une ressemblance générale frappante, une différenciation importante. Cet aspect de l'individualisation spécifique démontre qu'une connaissance approfondie de la morphologie de tous les appendices s'impose, celle-ci étant la seule qui puisse conduire à tirer au clair les rapports réels entre les divers taxa.

En considérant les péréiopodes VIII mâles, on voit, une fois de plus, que ces appendices sont les seuls à indiquer autant l'appartenance de $B$.(B.) vaducrisensis n.sp. au sous-genre Bathynella(Bathynella) et sa place à côté de B.(B.) boteai, que sa qualité d'espèce valide. Même si la chétotaxie des péréiopodes I-VII vient renforcer, par ses traits les plus importants, les deux dernières indications d'ordre systématique que nous offre l'étude des pénis, elle n'implique pas à elle seule, les mêmes résultats. Ce point de vue peut être aisément vérifié si l'on essaye d'élucider, seulement d'après la morphologie des femelles, la position systématique de l'espèce.

Pour mieux mettre en évidence les rapports morphologiques entre les deux taxa décrits, dans le tableau 8 on présente aussi bien les ressemblances que les différences entre leurs appendices.

\section{Bathynella(Bathynella) plesai Serban, 1971 (Pl. 16-21)}

Matériel. L'espèce a été décrite pour la première fois (Serban, 1971) d'après 2 mâles trouvés par C. Plesa dans la grotte "Pestera Closani", village Closani, dép. de Gorj, Olténie, 30. VIII. 1960. Onze ans après, C. Plesa retrouve l'espèce dans la même grotte, collectant un plus grand nombre d'individus (14.X.1971). Les données ci-dessous ont été obtenues en étudiant, non seulement le matériel de 1960, mais aussi les 5 mâles et 5 femelles provenant de la seconde capture.

Taille: $0,7 \mathrm{~mm}-0,85 \mathrm{~mm}$.

Antennule (Pl. 16A). De même que chez B.(B.) paranatans, l'article basal du pédoncule porte 2 poils sur sa face latéro-interne.

Antenne (Pl. 16B). Les articles II et IV de l'endopodite, de la même longueur, sont plus courts que le cinquième. L'exopodite est presque de la même longueur que l'article endopodial III; poil médian bien développé.

Mandibule (P1. 16C). Elle conserve la structure typique.

Maxillule (Pl. 16D). Dans la partie distale de cette pièce buccale on trouve 3 dents maxillulaires à épines et une dent glabre. Chez deux mâles, la dent médiane (la deuxième) de l'une des maxillules est munie de 3 épines.

Maxille (Pl. 16E). Les 5 poils du palpe sont des poils endopodiaux simples.

Péréiopodes I-VII (Pl. 17, 18, 21A). La paire I ne porte pas d'épipodite respiratoire; l'exite précoxal, vu de face, à contour semi-circulaire; éperon coxal des péréiopodes VII mâles, allongé. 


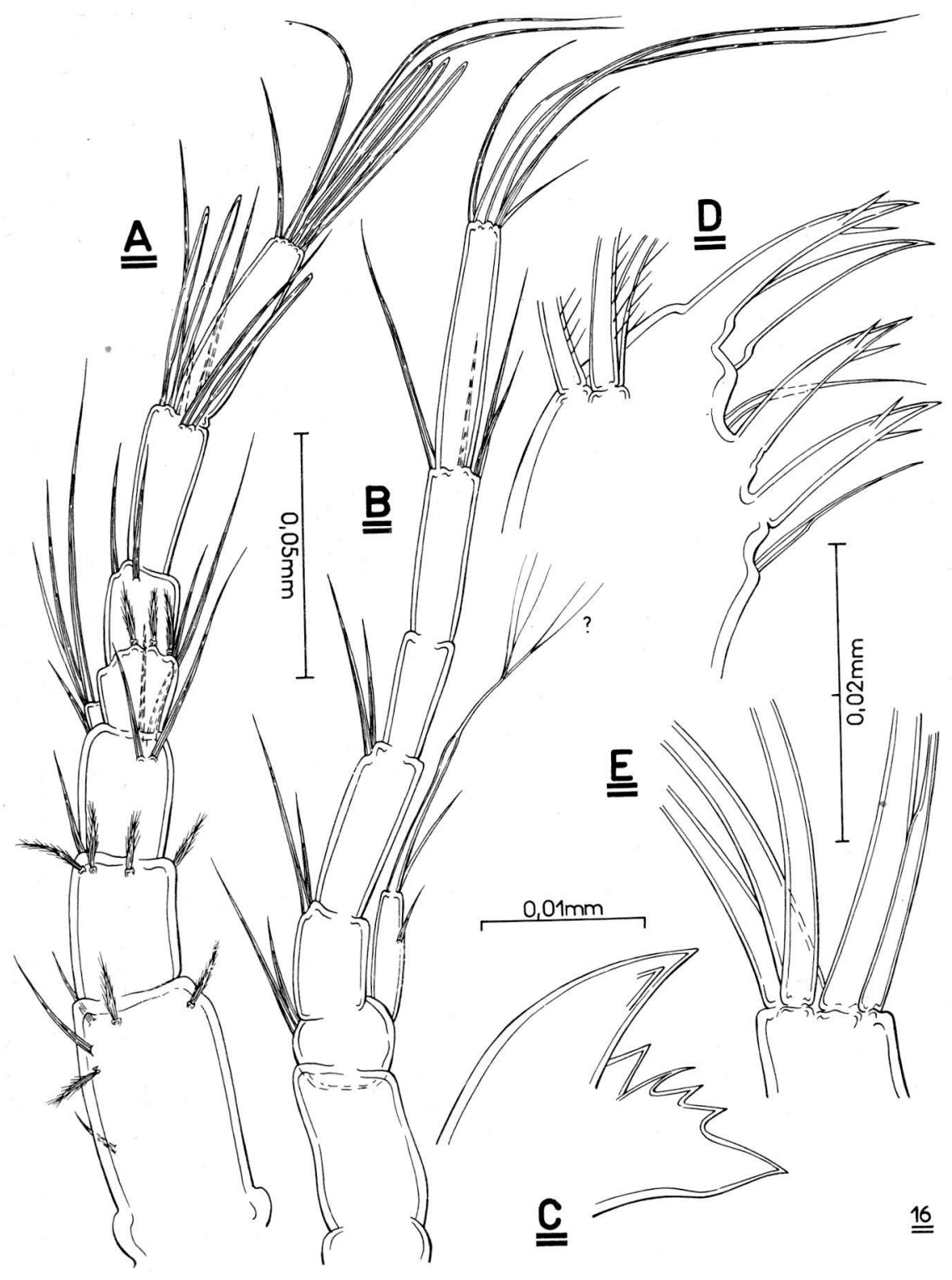

Planche 16. Bathynella (Bathynella) plesai Serban. A, antennule; B, antenne; C, partie masticatrice de la mandibule; $\mathrm{D}$, région distale de la maxillule; $\mathrm{E}$, les poils du palpe maxillaire. 


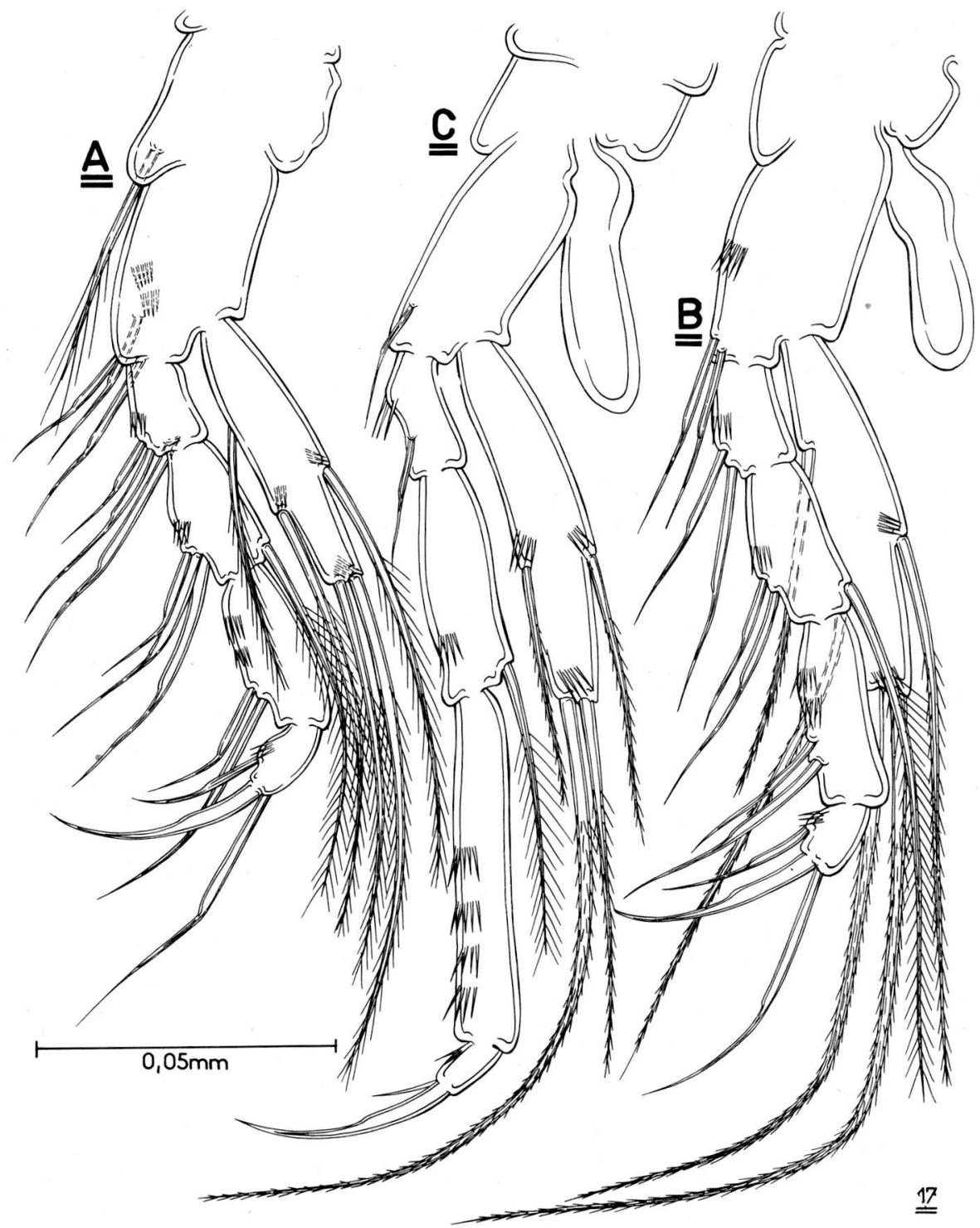

Planche 17. Bathynella (Bathynella) plesai Serban. A, péréiopode I; B, péréiopode II; C, péréiopode VII. 


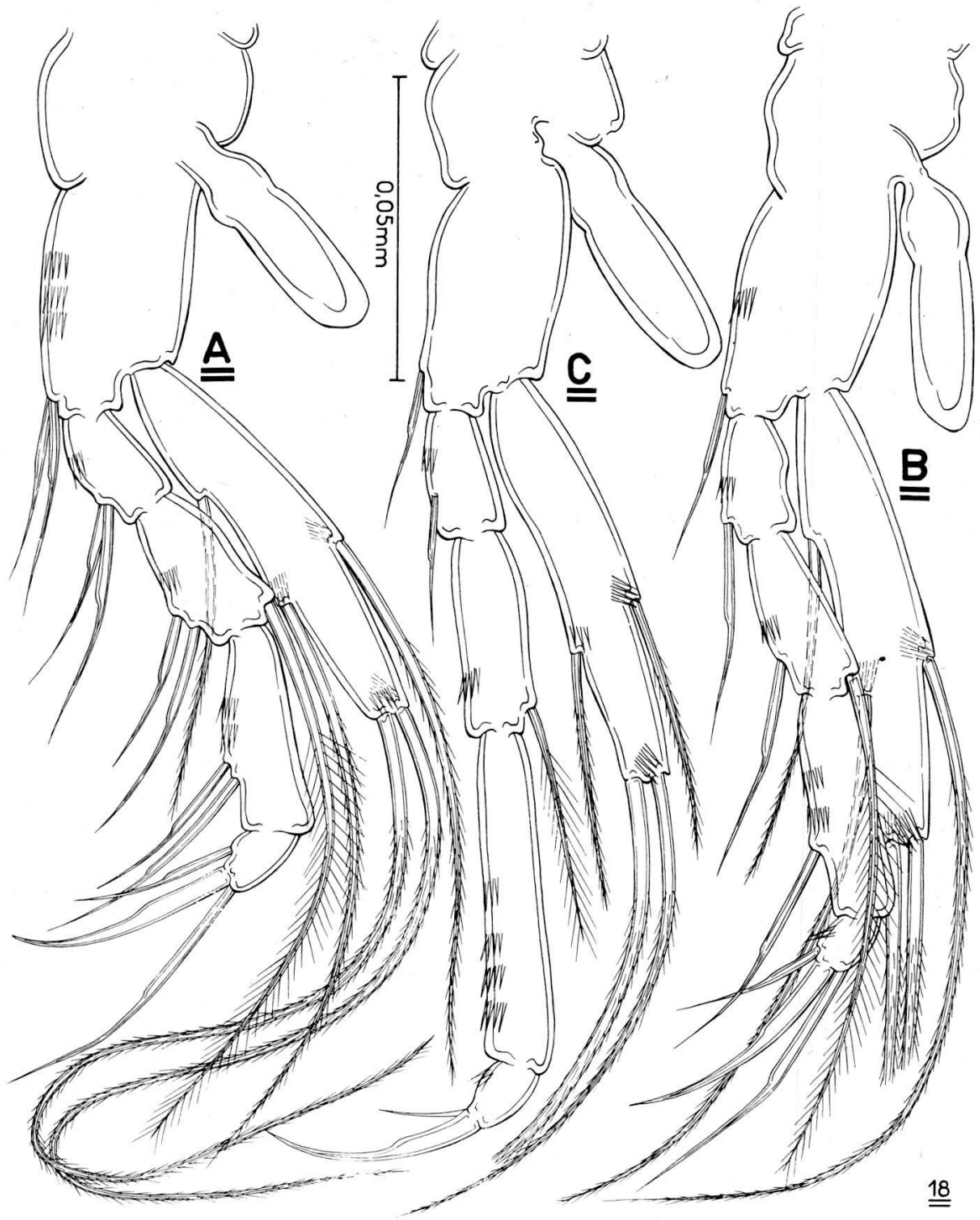

Planche 18. Bathynella (Bathynella) plesai Serban. A, péréiopode III; B, péréiopode V; C, péréiopode VI. 


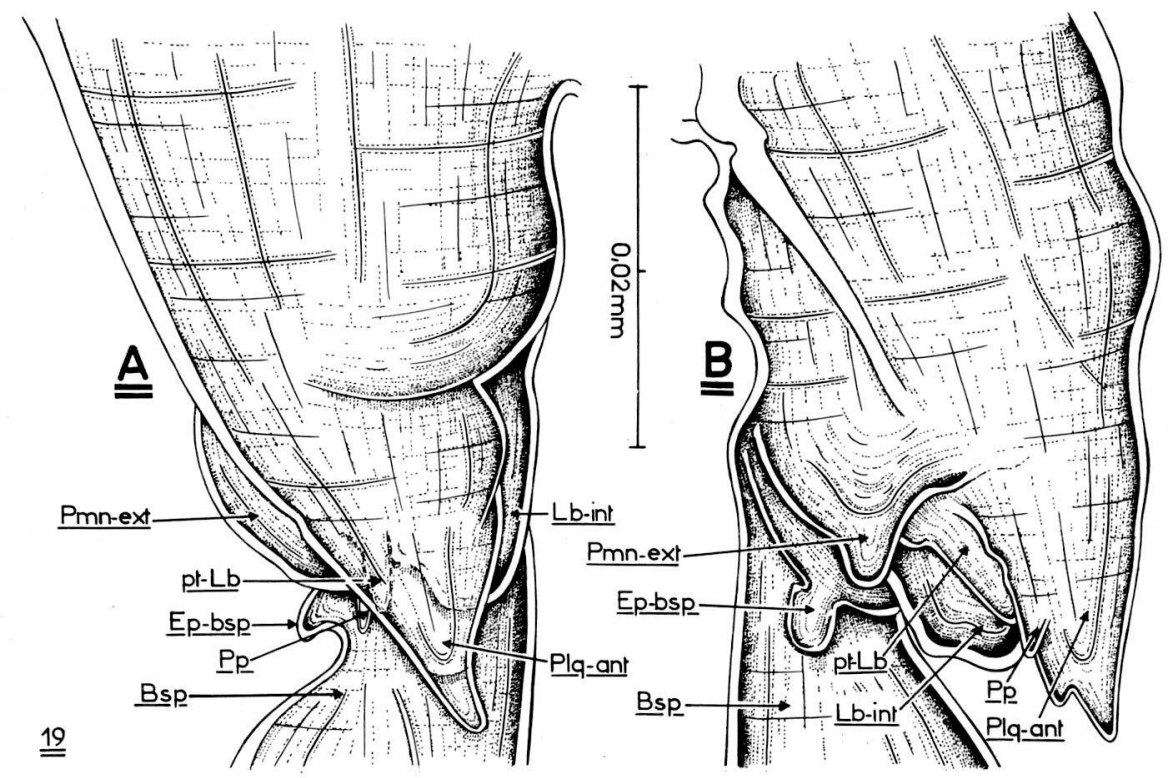

Planche 19. Bathynella (Bathynella) plesai Serban. Partie pénienne du péréiopode VIII mâle: A, face rostrale; B, face latéro-externe. Bsp, basipodite; Ep-bsp, éperon du basipodite; Lb-int, lobe interne; Plq-ant, plaque antérieure; Pmn-ext, proéminence externe; Pp, papille; $\mathbf{p t - L b}$,
petit lobe.

La chétotaxie, bien différente de celle des espèces B.(B.) boteai et B.(B.) vaducrisensis n. sp., ressemble généralement à celle de $B$.(B.) paranatans; toutefois, certaines caractéristiques que nous verrons plus bas ne sont pas à négliger:

a) autant chez les mâles (tableau 9) que chez les femelles (tableau 10), la chétotaxie des péréiopodes I est plus réduite que chez B.(B.) paranatans, étant composée de 40 et respectivement 42 poils; cette réduction numérique a lieu au niveau du premier article endopodial qui ne porte jamais plus de 3 poils;

b) la combinaison numérique fondamentale de la chétotaxie, 0/0 2/2 2/2 2/2 2/2 $3 / 35 / 5$, identique à celle de $B$. (B.) paranatans, est présente sur les péréiopodes II et III des deux sexes; ceci distingue l'espèce de $B$. (B.) paranatans dont les paires II-III des mâles et III-IV des femelles portent une chétotaxie correspondant à la combinaison numérique fondamentale;

c) bien que la chétotaxie des péréiopodes IV soit variable, les péréiopodes $\mathrm{V}$ des deux sexes ont toujours le même nombre de poils; la combinaison 0/0 1/1 1/1 1/1 $1 / 13 / 35 / 5$, comprenant 24 phanères, représente la chétotaxie la moins riche de ces appendices qui se rencontre chez les espèces du genre Bathynella;

d) vu l'identité de la combinaison numérique des péréiopodes $\mathrm{V}$ des deux sexes ainsi que celle des paires II et III, VI et VII, il ressort que chez B.(B.) plesai on 


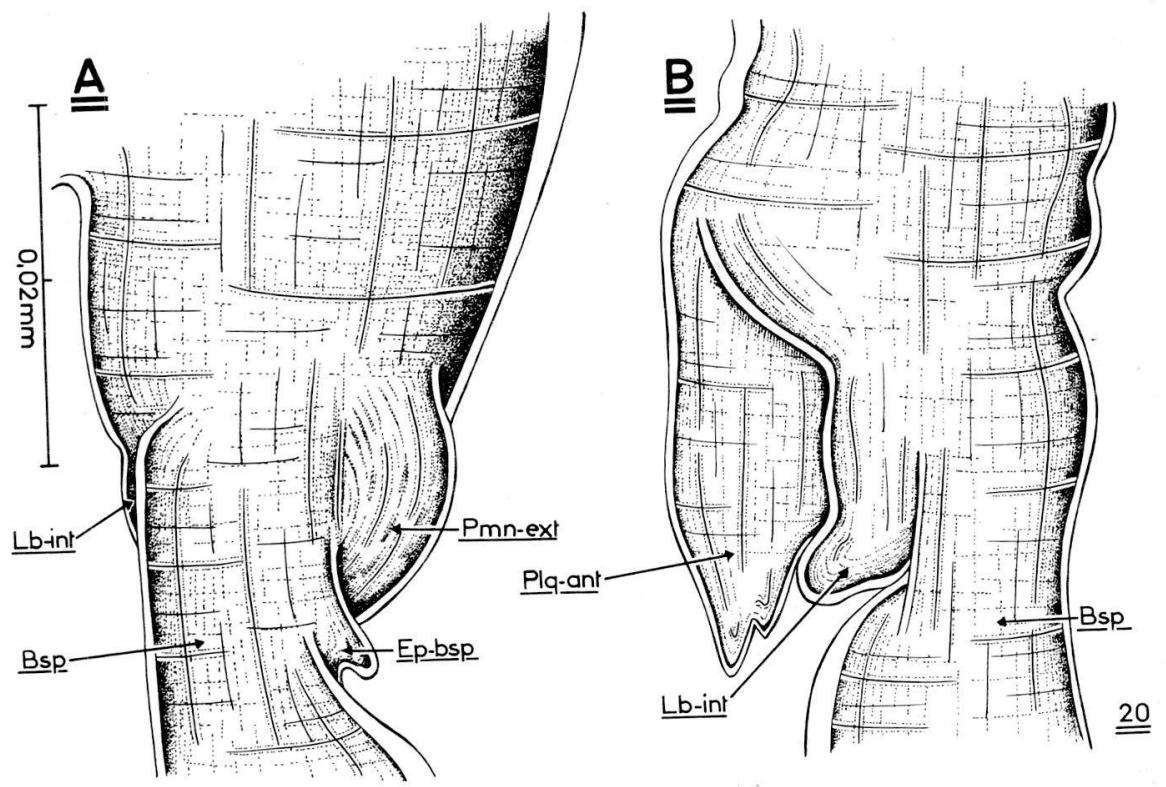

Planche 20. Bathynella (Bathynella) plesai Serban. Partie pénienne du péréiopode VIII mâle: A, face caudale; B, face latéro-interne. Bsp, basipodite; Ep-bsp, éperon du basipodite; Lb-int, lobe interne; Plq-ant, plaque antérieure; Pmn-ext, proéminence externe.

trouve 5 paires d'extrémités ambulatoires ayant la même chétotaxie chez les mâles que chez les femelles; rappelons que chez B.(B.) paranatans cette identité n'est valable que dans le cas des 3 paires - péréiopodes III, VI et VII;

e) comme il a été déja souligné (Serban, 1971), la chétotaxie des péréiopodes se caractérise aussi par les dimensions du phanère médian des articles apicaux des endopodites (paires I-V), visiblement plus robuste que les deux autres;

f) généralement, l'article endopodial III des paires VI et VII porte 4 cténidies;

g) enfin, une dernière remarque quant au nombre bien variable des poils exopodiaux des péréiopodes VI: dans le cas de 3 exemplaires, nous avons trouvé 4 poils sur un appendice et 5 sur l'autre, chez 4 individus, 4 poils sur les deux appendices, tandis que chez 3 autres, 5 poils.

Péréiopode VIII mâle (PI. 19, 20). La plaque antérieure (Plq-ant) se termine par 2 prolongements pointus; le prolongement qui se trouve dans la partie rostrale, trois fois plus long que le prolongement caudal (Pl. 19B, 20B), a une largeur basale qui dépasse les $2 / 3$ de la partie de la plaque qui le porte. Vue de face, la plaque antérieure prend un aspect triangulaire, sa région apicale étant pointue (région qui correspond au prolongement rostral). Le petit lobe (pt-Lb) est allongé. La proéminence externe (Pmn-ext) a le bord semi-circulaire (Pl. 19A), 20A). 
Tableau 9. Bathynella (Bathynella) plesai Serban

Combinaisons numériques de la chétotaxie des péréiopodes I-VII (mâle)

\begin{tabular}{lcccccccc}
\hline Péréiopode & Cx & Bsp & I & II & III & IV & Exp & $\begin{array}{c}\text { Nombre total } \\
\text { de poils }\end{array}$ \\
\hline I & $1 / 1$ & $3 / 3$ & $3 / 3$ & $3 / 3$ & $2 / 2$ & $3 / 3$ & $5 / 5$ & 40 \\
II & $0 / 0$ & $2 / 2$ & $2 / 2$ & $2 / 2$ & $2 / 2$ & $3 / 3$ & $5 / 5$ & 32 \\
III & $0 / 0$ & $2 / 2$ & $2 / 2$ & $2 / 2$ & $2 / 2$ & $3 / 3$ & $5 / 5$ & 32 \\
IV & $0 / 0$ & $1 / 1$ & $2 / 2$ & $2 / 2$ & $1 / 1$ & $3 / 3$ & $5 / 5$ & 28 \\
V & $0 / 0$ & $1 / 1$ & $1 / 1$ & $1 / 1$ & $1 / 1$ & $3 / 3$ & $5 / 5$ & 24 \\
VI & $0 / 0$ & $1 / 1$ & $1 / 1$ & $0 / 0$ & $0 / 0$ & $2 / 2$ & $5 / 5$ & 18 \\
VII & $0 / 0$ & $1 / 1$ & $1 / 1$ & $0 / 0$ & $0 / 0$ & $2 / 2$ & $4 / 4$ & 16
\end{tabular}

Tableau 10. Bathynella (Bathynella) plesai Serban

Combinaisons numériques de la chétotaxie des péréiopodes I-VII (femelle)

\begin{tabular}{lcccccccc}
\hline Péréiopode & Cx & Bsp & I & II & III & IV & Exp & $\begin{array}{c}\text { Nombre total } \\
\text { de poils }\end{array}$ \\
\hline I & $1 / 1$ & $3 / 3$ & $3 / 3$ & $3 / 3$ & $3 / 3$ & $3 / 3$ & $5 / 5$ & 42 \\
II & $0 / 0$ & $2 / 2$ & $2 / 2$ & $2 / 2$ & $2 / 2$ & $3 / 3$ & $5 / 5$ & 32 \\
III & $0 / 0$ & $2 / 2$ & $2 / 2$ & $2 / 2$ & $2 / 2$ & $3 / 3$ & $5 / 5$ & 32 \\
IV & $0 / 0$ & $1 / 1$ & $2 / 2$ & $2 / 2$ & $2 / 2$ & $3 / 3$ & $5 / 5$ & 30 \\
V & $0 / 0$ & $1 / 1$ & $1 / 1$ & $1 / 1$ & $1 / 1$ & $3 / 3$ & $5 / 5$ & 24 \\
VI & $0 / 0$ & $1 / 1$ & $1 / 1$ & $0 / 0$ & $0 / 0$ & $2 / 2$ & $5 / 5$ & 18 \\
VII & $0 / 0$ & $1 / 1$ & $1 / 1$ & $0 / 0$ & $0 / 0$ & $2 / 2$ & $4 / 4$ & 16
\end{tabular}

Péréiopode VIII femelle (Pl. 21B). L'exopodite est plus long que le basipodite et l'endopodite pris ensemble. Les deux poils de ses bords sont situés à la limite distale du tiers médian de la rame.

Pléopode ( $\mathrm{Pl} .21 \mathrm{C}$ ). L'article distal du pléopode est pourvu, pareillement à celui de $B$. (B.) paranatans, de 5 poils.

Uropode (Pl. 21D). Le sympodite porte 6-7 épines. La griffe terminale de l'endopodite, plus longue que la rame, est d'un tiers de sa longueur pius développée que la griffe médiane; la griffe proximale est deux fois plus courte que la griffe distale. 


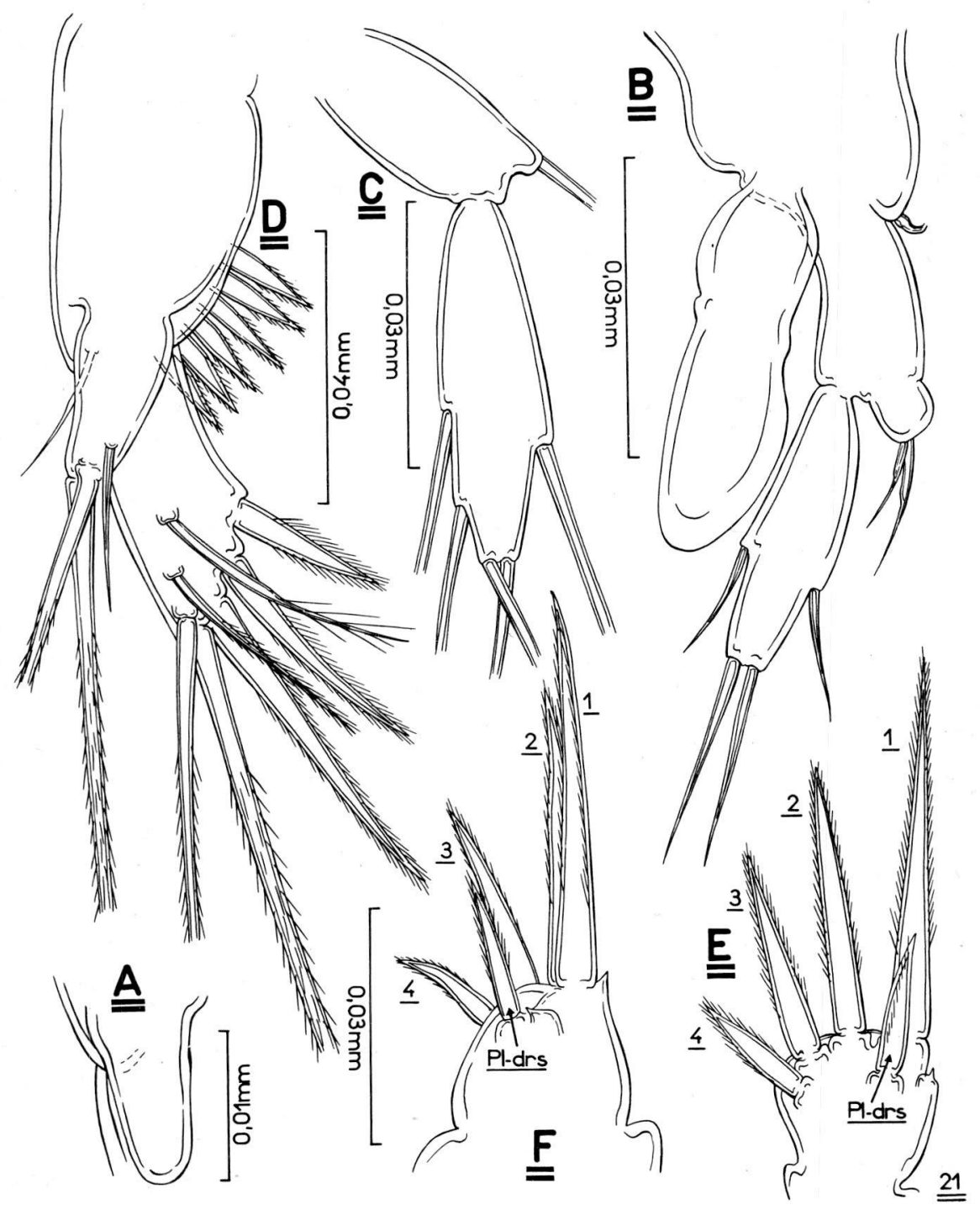

Planche 21. Bathynella (Bathynella) plesai Serban. A, éperon coxal du péréiopode VII mâle; $\mathrm{B}$, péréiopode VIII femelle; $\mathrm{C}$, pléopode; $\mathrm{D}$, uropode; $\mathrm{E}$, furca, face dorsale; $\mathrm{F}$, furca, face latéro-externe. 1,2,3,4, poils apicaux de la furca; PI-drs, poil dorsal. 
Tableau 11. Bathynella (Bathynella) plesai Serban et

\begin{tabular}{|c|c|c|}
\hline Caractère & $\frac{\text { B.(B.) plesai }}{\text { Serban }}$ & $\frac{\text { B.(B.) ruffoi }}{\text { Serban }}$ \\
\hline antenne & - exopodite à poil médian & $\begin{array}{l}\text { - exopodite sans poil } \\
\text { médian }\end{array}$ \\
\hline $\begin{array}{c}\text { péréiopodes } \\
\text { I-VII }\end{array}$ & $\begin{array}{l}\text { - } \mathrm{P}_{\text {I }} \text { sans épipodite respi- } \\
\text { ratoire } \\
\text { - poil médian de l'article } \\
\text { IV }\left(\mathrm{P}_{\mathrm{I}}-\mathrm{P}_{\mathrm{V}}\right) \text { de taille bien } \\
\text { plus robuste que les } \\
\text { autres } \\
\text { - } \mathrm{P}_{\mathrm{V}} \text { à } 4 \text { poils exopodiaux }\end{array}$ & $\begin{array}{l}\text { - } \mathrm{P}_{\mathrm{I}} \text { à épipodite respi- } \\
\text { ratoire } \\
\text { - poil médian de l'arti- } \\
\text { cle IV }\left(\mathrm{P}_{I^{-}}-\mathrm{P}_{\mathrm{V}}\right) \text { d'une } \\
\text { robustesse moins } \\
\text { accentueé } \\
\text { - } \mathrm{P}_{\mathrm{VII}} 5 \text { poils exopodiaux }\end{array}$ \\
\hline $\begin{array}{l}\text { péréiopode } \\
\text { vIII mâle }\end{array}$ & $\begin{array}{l}\text { - plaque antérjeure à } 2 \\
\text { prolongements de taille } \\
\text { bien différente } \\
\text { - partie apicale des pro- } \\
\text { longements, pointue }\end{array}$ & $\begin{array}{l}\text { - plaque antérieure à } 2 \\
\text { prolongements de taille } \\
\text { moins différente } \\
\text { - partie apicale des pro- } \\
\text { longements, arrondie }\end{array}$ \\
\hline pléopode & - article distal à 5 poils & - article distal à 4 poils \\
\hline furca & $\begin{array}{l}\text { - poil } 1 \text { plus long que le } \\
\text { poil } 2 \\
\text { - poil dorsal, } 2,5 \text { fois } \\
\text { plus court que le poil } 1\end{array}$ & $\begin{array}{l}\text { - poil l égal au poil } 2 \\
\text { - poil dorsal, } 3 \text { fois plus } \\
\text { court que le poil } 1\end{array}$ \\
\hline
\end{tabular}

Furca (Pl. 21E, F). Lors de la première description de l'espèce (Serban, 1971), il a été précisé que la furca se caractérise par la réduction graduelle de la longueur des poils 1-4. L'étude du nouveau matériel permet de mentionner aussi d'autres traits: le poil 1, deux fois et demie plus long que le bras furcal, dépasse d'un quart de sa longueur le poil 2; le poil 3 atteint les $4 / 5$ de la longueur du précédent; le poil dorsal, de même taille que le poil 4, est moins de trois fois plus court que le poil 1 .

Diagnose. Plaque antérieure du péréiopode VIII mâle finissant par 2 prolongements pointus; prolongement rostral, plus développé que le prolongement caudal; proéminence externe à bord arrondi, semi-circulaire. Péréiopodes I à 40-42 poils; combinaison numérique fondamentale de la chétotaxie, $0 / 0$ 2/2 2/2 2/2 2/2 3/3 $5 / 5$, présente sur les paires II et III des deux sexes; les péréiopodes $\mathrm{V}$, à chétotaxie identique dans les deux sexes, portent 24 poils terminaux; paire I-V à 3 poils; poil 
médian visiblement plus robuste; exopodite des péréiopodes VII à 4 poils. Article distal du pléopode avec 5 poils. Uropode muni de 6-7 épines sur le sympodite et de 3 griffes endopodiales. Poils furcaux 14 de tailles qui se réduisent graduellement; poil dorsal, de longueur égale au poil 4 , est moins de trois fois plus court que le poil 1. Antennule, maxillule et maxille pareilles à celles de B.(B.) paranatans.

\section{Remarques}

Selon la plupart des caractères, B.(B.) plesai est plus proche de B.(B.) paranatans que de $B$.(B.) boteai ou de l'espèce nouvelle $B$.(B.) vaducrisensis $\mathrm{n}$. sp. Mais les péréiopodes VIII mâles, la furca et la chétotaxie des péréiopodes distinguent nettement ce taxon de B.(B.) paranatans.

Un problème qui mérite d'être posé est celui des rapports morphologiques entre B.(B.) plesai Serban de Roumanie et B.(B.) ruffoi Serban d'Italie.

Grâce à l'amabilité de M. Sandro Ruffo du Museo Civico di Storia Naturale, Verona, nous avons eu la chance d'étudier certains matériaux provenant de la Lombardie (Serban, 1973). Nous avons décrit deux espèces nouvelles, B.(B.) ruffoi et B.(B.) lombardica, en instituant pour la seconde un nouveau sous-genre, Bathynella(Lombardobathynella). A la suite de ces recherches, les Bathynelles de la Péninsule sont représentés par deux taxa appartenant au genre Bathynella auxquels s'ajoute l'espèce stammeri (Delamare Deboutteville, 1960); selon nous, cette dernière doit être tenue comme le représentant d'un genre indépendant, Antrobathynella Serban (Serban, 1973a). En considérant la morphologie du pénis, l'espèce ruffoi s'avère proche de $B$.(B.) plesai, ces deux taxa étant les seuls à avoir les plaques antérieures munies de deux prolongements apicaux. En partant de la nouvelle description de B.(B.) plesai et pour mieux souligner les relations entre celui-ci et $B$.(B.) ruffoi, dans le tableau 11 on a donné les caractères les plus importants qui séparent les deux taxa. Comme on le voit, en dehors de la différenciation de la furca, des péréiopodes I et du péréiopode VIII mâle, on trouve aussi d'autres traits distinctifs, comme la chétotaxie de l'exopodite antennaire, des péréiopodes et du pléopode.

\section{Bathyriella (Bathynella) motrensis Serban, 1971 (P1. 22-26)}

Materièl. Les 10 individus étudiés proviennent du milieu interstitiel de la rivière de Motru Mare, village Closani, dép. de Gorj, Olténie, 15,IV.1959, 15.X.1960, 20.VIII.1967.

Taille: environ $1 \mathrm{~mm}$.

Antennule (Pl. 22A). L'article basal du pédoncule porte les 2 poils de la face latéro-interne.

Antenne (Pl. 22B). L'article endopodial II, de la même taille que le quatrième, est plus court que l'article V. L'exopodite, bien développé, dépasse la longueur de l'article endopodial III.

Mandibule (P1. 22C). Sa partie masticatrice ne présente pas de différences par rapport à celle des mandibules de $B$.(B.) paranatans et $B$. (B.) plesai.

Maxillule (P1. 22D). Elle est identique à celle de B.(B.) paranatans. 


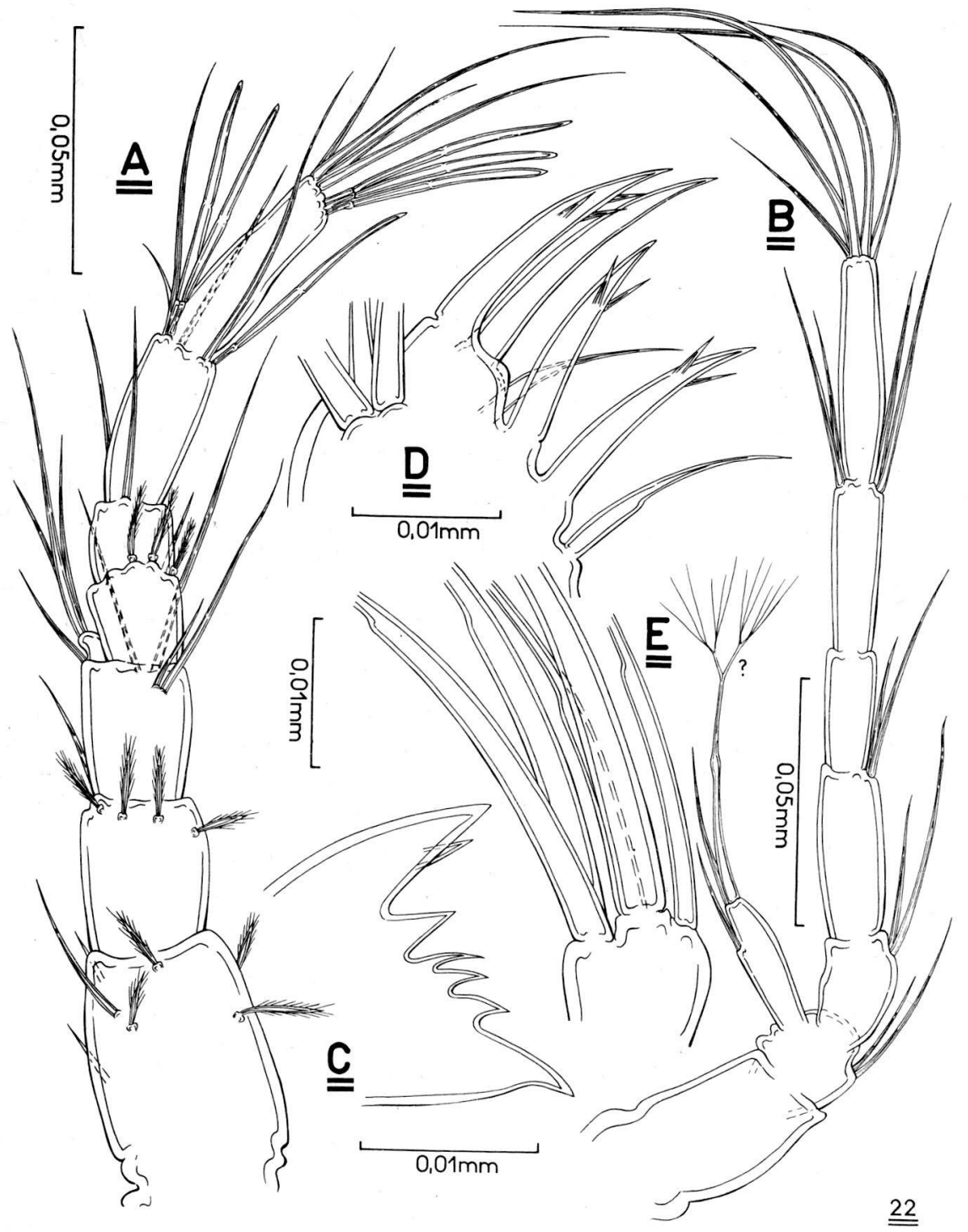

Planche 22. Bathynella (Bathynella) motrensis Serban. A, antennule; B, antenne; C, partie masticatrice de la mandibule; $\mathrm{D}$, région distale de la maxillule; $\mathrm{E}$, les poils du palpe maxillaire. 
Maxille (Pl. 22E). Les 5 phanères du palpe sont des poils endopodiaux simples.

Péréiopodes I-VII (P1. 23). L'épipodite respiratoire n'est pas développé sur les péréiopodes I; l'exite précoxal à bord arrondi. La chétotaxie ressemble à celle de B.(B.) paranatans et B.(B.) plesai; elle présente les particularités suivantes (tableaux 12 et 13$)$ :

a) par le nombre et la répartition des poils, la chétotaxie des péréiopodes I des mâles rappelle $B$.(B.) plesai, tandis que celle des mêmes appendices femelles, B.(B.) paranatans;

Tableau 12. Bathynella (Bathynella) motrensis Serban

Combinaisons numériques de la chétotaxie des péréiopodes I-VII (mâle)

\begin{tabular}{lcccccccc}
\hline Péréiopode & Cx & Bsp & I & II & III & IV & Exp & $\begin{array}{c}\text { Nombre total } \\
\text { de poils }\end{array}$ \\
\hline I & $1 / 1$ & $3 / 3$ & $3 / 3$ & $3 / 3$ & $2 / 2$ & $3 / 3$ & $5 / 5$ & 40 \\
II & $0 / 0$ & $2 / 2$ & $2 / 2$ & $2 / 2$ & $2 / 2$ & $3 / 3$ & $5 / 5$ & 32 \\
III & $0 / 0$ & $2 / 2$ & $2 / 2$ & $2 / 2$ & $2 / 2$ & $3 / 3$ & $5 / 5$ & 32 \\
IV & $0 / 0$ & $1 / 1$ & $2 / 2$ & $2 / 2$ & $1 / 1$ & $3 / 3$ & $5 / 5$ & 28 \\
V & $0 / 0$ & $1 / 1$ & $2 / 2$ & $1 / 1$ & $1 / 1$ & $3 / 3$ & $5 / 5$ & 26 \\
VI & $0 / 0$ & $1 / 1$ & $1 / 1$ & $0 / 0$ & $0 / 0$ & $2 / 2$ & $5 / 5$ & 18 \\
VII & $0 / 0$ & $1 / 1$ & $1 / 1$ & $0 / 0$ & $0 / 0$ & $2 / 2$ & $4 / 4$ & 16
\end{tabular}

Tableau 13. Bathynella (Bathynellal motrensis Serban Combinaisons numériques de la chétotaxie des péréiopodes I-VII (femelle)

\begin{tabular}{lcccccccc}
\hline Péréiopode & Cx & Bsp & I & II & III & IV & Exp & $\begin{array}{c}\text { Nombre total } \\
\text { de poils }\end{array}$ \\
\hline I & $1 / 1$ & $3 / 3$ & $4 / 4$ & $3 / 3$ & $3 / 3$ & $3 / 3$ & $5 / 5$ & 44 \\
II & $0 / 0$ & $2 / 2$ & $2 / 2$ & $2 / 2$ & $2 / 2$ & $3 / 3$ & $5 / 5$ & 32 \\
III & $0 / 0$ & $2 / 2$ & $2 / 2$ & $2 / 2$ & $2 / 2$ & $3 / 3$ & $5 / 5$ & 32 \\
IV & $0 / 0$ & $2 / 2$ & $2 / 2$ & $2 / 2$ & $2 / 2$ & $3 / 3$ & $5 / 5$ & 32 \\
V & $0 / 0$ & $1 / 1$ & $2 / 2$ & $2 / 2$ & $1 / 1$ & $3 / 3$ & $5 / 5$ & 28 \\
VI & $0 / 0$ & $1 / 1$ & $1 / 1$ & $0 / 0$ & $0 / 0$ & $2 / 2$ & $5 / 5$ & 18 \\
VII & $0 / 0$ & $1 / 1$ & $1 / 1$ & $0 / 0$ & $0 / 0$ & $2 / 2$ & $4 / 4$ & 16
\end{tabular}




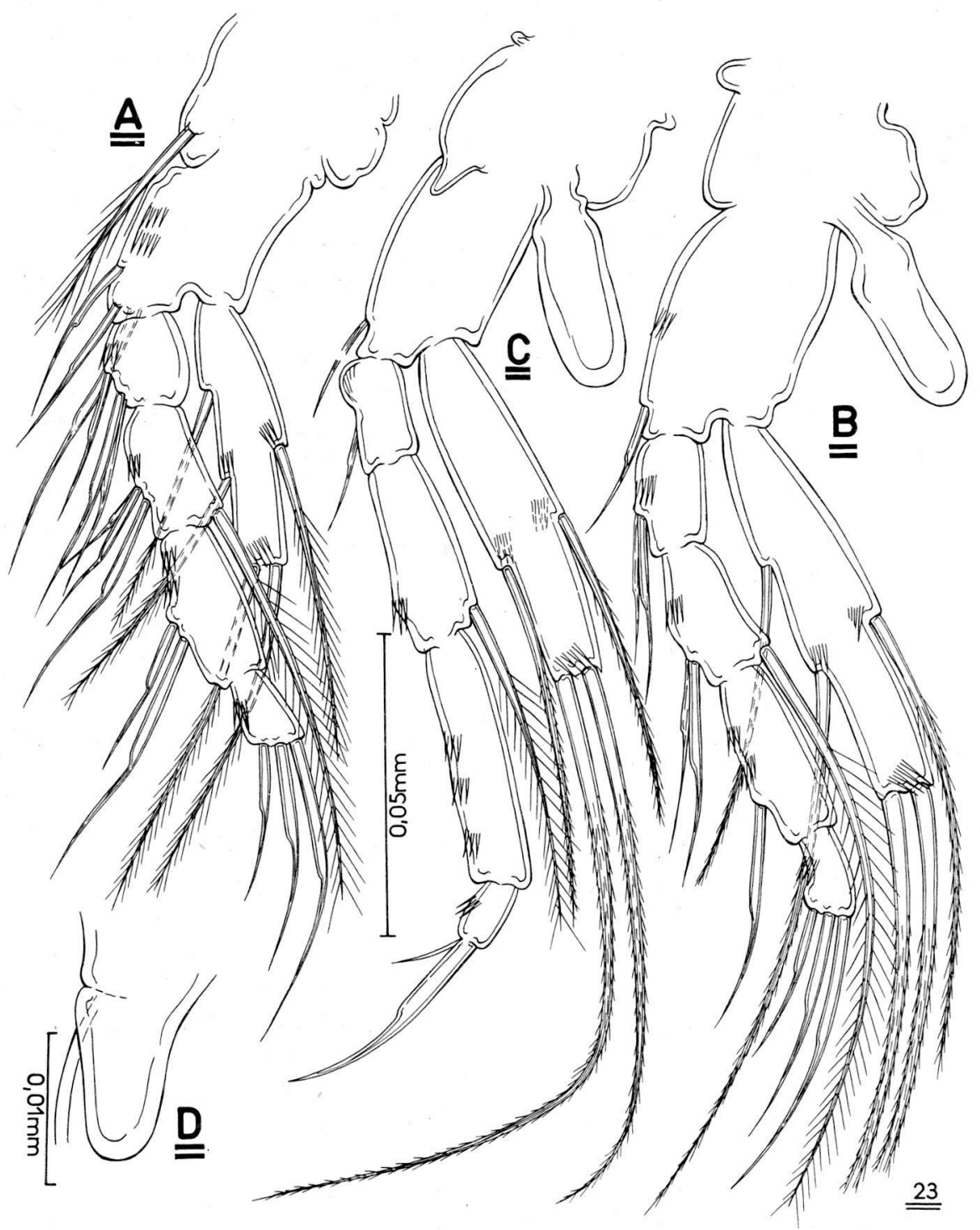

Planche 23. Bathynella (Bathynella) motrensis Serban. A, péréiopode I; B, péréiopode V; $\mathrm{C}$, péréiopode VII; D, éperon coxal du péréiopode VII mâle. 


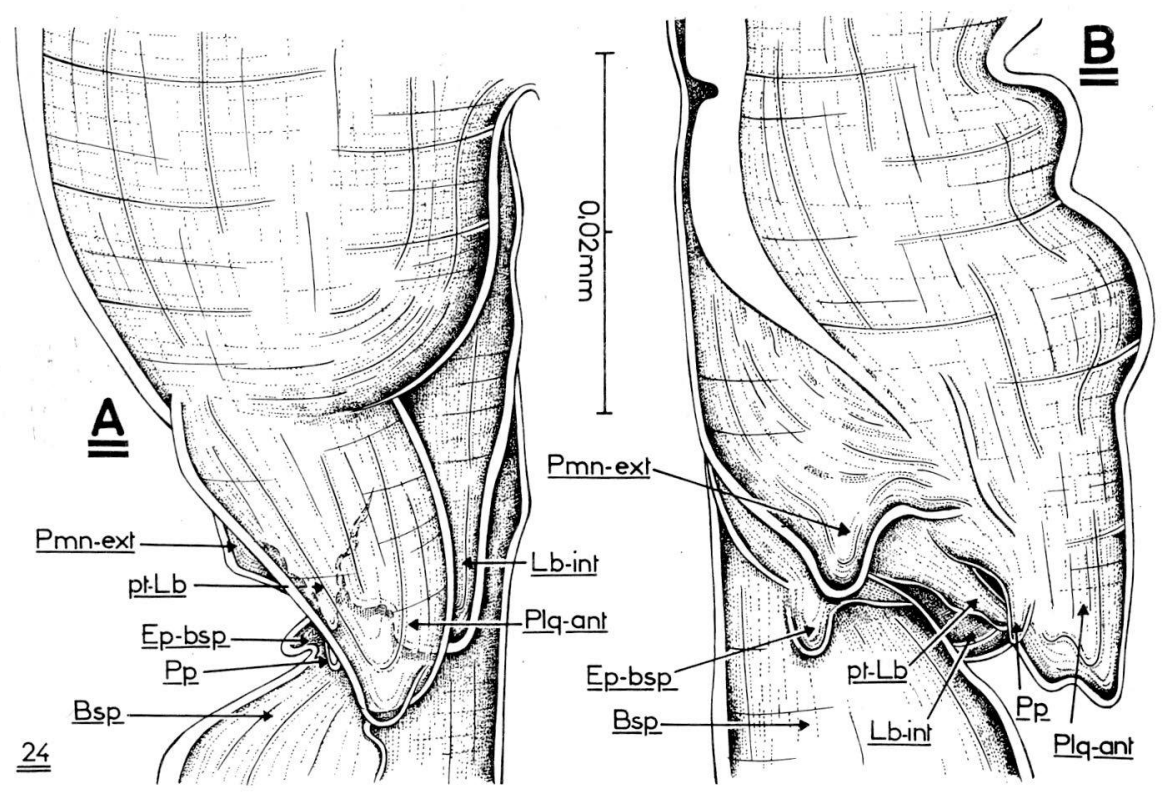

Planche 24. Bathynella (Bathynella) motrensis Serban. Partie pénienne du péréiopode VIII mâle: A, face rostrale; B, face latéro-externe. Bsp, basipodite; Ep-bsp, éperon du basipodite; Lb-int, lobe interne; Plq-ant, plaque antérieure; Pmn-ext, proéminence externe; Pp, papille; pt-Lb, petit lobe.

b) la combinaison numérique fondamentale - $0 / 02 / 2 \quad 2 / 2 \quad 2 / 2 \quad 2 / 2 \quad 3 / 3 \quad 5 / 5$-, identique à celle des espèces mentionnées, est présente sur. les péréiopodes II et III des mâles et II-IV des femelles. Si l'existence de cette combinaison sur les péréiopodes II des deux sexes, d'une part, et sur les péréiopodes IV des femelles, d'autre part, est une caractéristique qui se trouve chez B.(B.) plesai et respectivement chez B.(B.) paranatans, sa présence, chez les femelles, sur trois paires de péréiopodes, reste un trait qui ne se rencontre que chez B.(B.) boteai;

c) l'exopodite des paires VII est muni de 4 poils;

d) l'article' endopodial III des péréiopodes VI et VII porte 3 cténidies.

Péréiopode VIII mâle (Pl. 24, 25). La face rostrale de la plaque antérieure (Plqant; Pl. 24A), d'aspect similaire à celle de B.(B.) paranatans. La partie apicale de la plaque a deux mamelons; le mamelon rostral est plus développé que le mamelon caudal. La proéminence externe (Pmn-ext), généralement à contour arrondi, présente toutefois un angle dans la région où les parties verticale et horizontale du bord entrent en connexion. Chez B.(B.) paranatans et B.(B.) plesai, le bord est parfaitement semi-circulaire. 


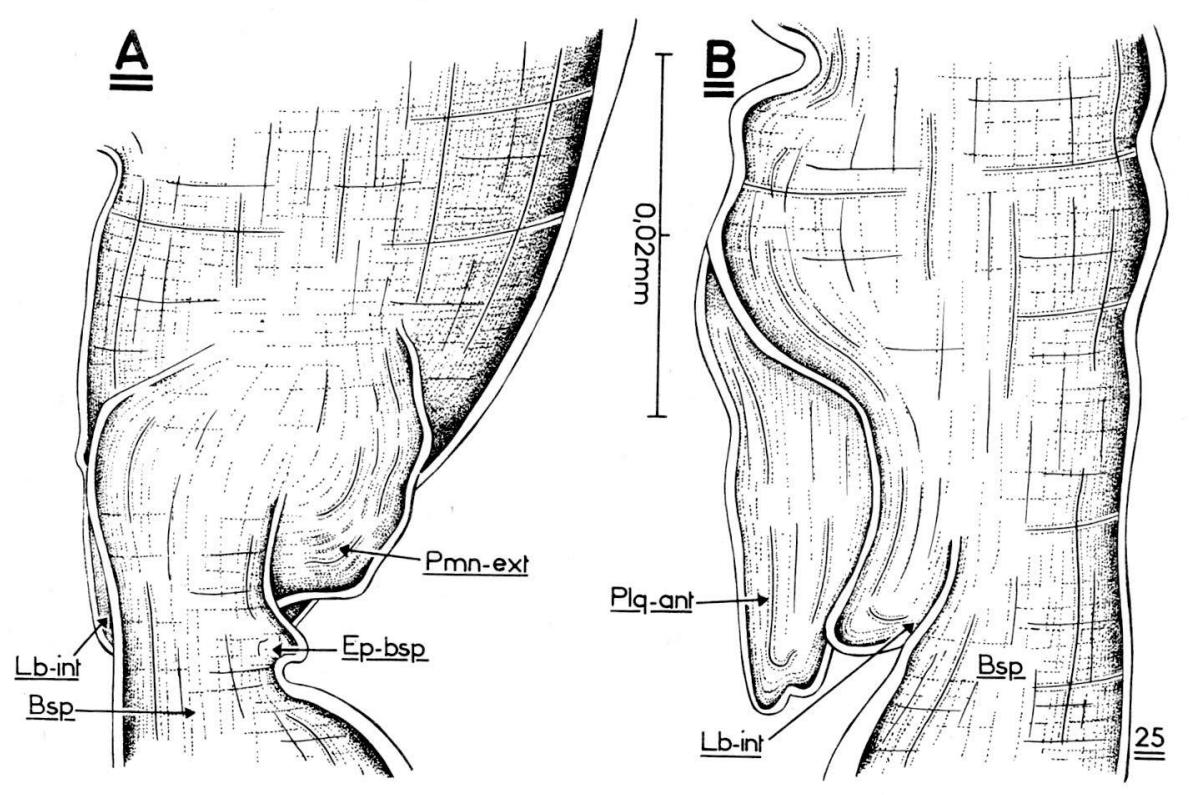

Planche 25. Bathynella (Bathynella) motrensis Serban. Partie pénienne du péréiopode VIII mâle: A, face caudale; B, face latéro-interne. Bsp, basipodite; Ep-bsp, éperon du basipodite; Lb-int, lobe interne; Plq-ant, plaque antérieure; Pmn-ext, proéminence externe.

Péréiopode VIII femelle (Pl. 26A). Chez toutes les femelles étudiées, cet appendice se remarque par l'allongement de l'exopodite, 1,5 fois plus long que le basipodite et l'endopodite pris ensemble.

Pléopode (Pl. 26B). Article distal à 5 poils.

Uropode (Pl. 26C). Le sympodite porte toujours 5 épines. La griffe distale, une fois et demie plus longue que l'endopodite, dépasse respectivement d'environ deux et trois fois la longueur de la griffe médiane et de la griffe proximale.

Furca (Pl. 26E,D). Poils 1 et 2, de même taille; le poil 3, atteignant deux tiers de la longueur des premiers, est deux fois plus long que le poil 4 . Le poil dorsal, un peu plus de deux fois et demie plus court que le poil 1 , est égal au poil 4.

Diagnose. La plaque antérieure du péréiopode VIII mâle porte 2 mamelons apicaux; mamelon rostral mieux développé; la proéminence externe, à bord arrondi, présente un angle dans sa région ventrale. La chétotaxie des péréiopodes se caractérise par la présence de la combinaison numérique fondamentale $0 / 0$ 2/2 $2 / 22 / 22 / 23 / 35 / 5$ sur les paires II-III des mâles et II-IV des femelles; article endopodial IV des paires I-V à 3 poils; exopodite des péréiopodes VII avec 4 poils. Exopodite des péréiopodes VIII femelles, deux fois et demie plus long que le basipodite et l'endopodite pris ensemble. Article distal du pléopode muni de 5 phanères. Poils furcaux 1 et 2 de taille semblable; le poil dorsal, de la même longueur que le poil 4 , est un peu plus de deux fois et demie plus court que le poil 1. 


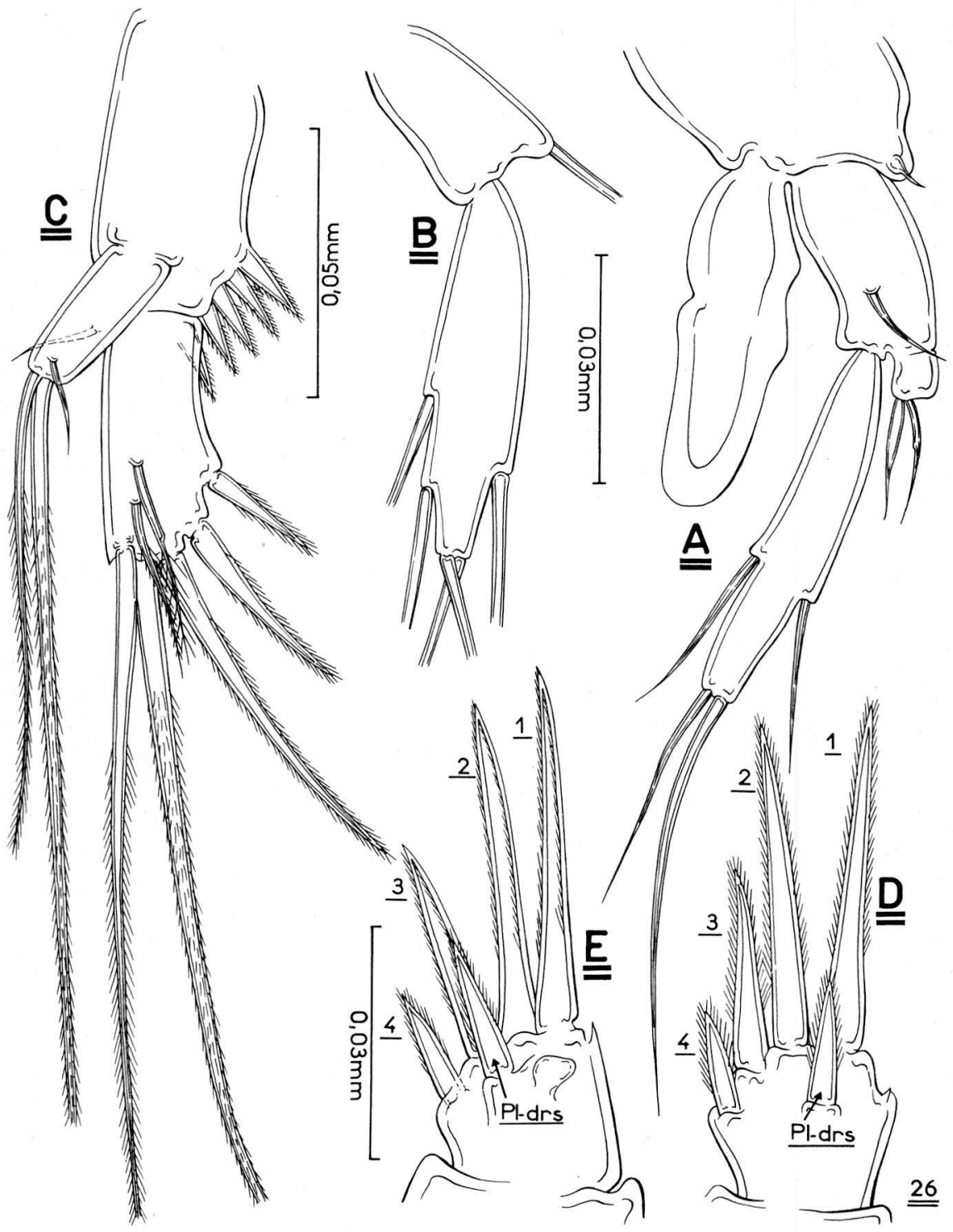

Planche 26. Bathynella (Bathynella) motrensis Serban. A, péréiopode VIII femelle; B, pléopode; $C$, uropode; $D$, furca, face dorsale; $E$, furca, face latéro-externe. $1,2,3,4$, poils apicaux de la furca; Pl-drs, poil dorsal. 


\section{Remarques}

Parmi les quatre espèces décrites par nous en 1971, c'est B.(B.) motrensis qui a soulevé les plus nombreuses incertitudes en ce qui concerne sa validité; sa ressemblance générale avec B.(B.) paranatans (voir antennule, maxillule, maxille, chétotaxie des péréiopodes et la furca) et surtout la similitude des péréiopodes VIII mâles, furent des éléments présupposant son statut infraspécifique. Mais, compte tenu des données très fragmentaires que nous possédions sur les Bathynella de Roumanie et surtout celles concernant leur répartition sur le territoire de notre pays, il nous a été impossible de lui conférer ce statut. Lors des dernières recherches faites avant d'achever l'étude intitulée "Bathynella" (Serban, 1972), nous avons mis en évidence un autre élément structural soutenant la validité de l'espèce B.(B.) motrensis. Il s'agit du dessin argyrophile de la cuticule qui, d'après les sclérites du pléon, est bien différent de celui de B.(B.) paranatans (voir les planches XIX, XX et LXII in Serban, 1972). Ce nouveau caractère, de même que l'absence de l'épipodite respiratoire sur les péréiopodes I, la chétotaxie des appendices ambulatoires, la forme de la plaque antérieure et de la proéminence externe du pénis et le nombre des épines sur le sympodite des uropodes prouvent que B.(B.) motrensis doit être tenu comme une espèce indépendante par rapport à $B$. (B.) paranatans.

\section{RESUME}

Dans cette première partie de la note on présente la description détaillée des espèces B.(B.) boteai Serban, B.(B.) motrensis Serban et B.(B.) plesai Serban dont les diagnoses préliminaires ont été publiées en 1971; une espèce nouvelle, B.(B.) vaducrisensis n. sp., est également décrite. A l'heure actuelle, le genre Bathynella Vejdovsky est représenté dans la faune de la Roumanie par 6 taxa, aux quatre espèces déjà mentionnées s'ajoutant B.(B.) paranatans Serban et B.(B.) scythica Botosaneanu et Damian.

N.B. La bibliographie sera donnée à la fin de la deuxième partie de ce travail. 